\title{
High shear rheometry using hydrodynamic lubrication flows
}

\author{
CHRISTIAN CLASEN
}

Department of Chemical Engineering, University of Leuven (KU Leuven), W. de Croylaan 46, 3001 Heverlee, Belgium

\section{Abstract}

In this paper we present an analytical solution of Reynolds' equation for the hydrodynamic lubrication flow in a parallel plate-on-annulus configuration of a conventional rotational rheometer. In this triborheometrical configuration originally proposed by Kavehpour and McKinley (Tribology Letters 17, 327 (2004)) the shearing surfaces are pushed together by an externally applied normal force. Our solution predicts quantitatively how lubrication forces are enlarging the gap $h$ between the surfaces with increasing angular velocity $\Omega$, depending solely on the unavoidable misalignment $\alpha$ of the surfaces. The predicted gap evolution as well as the predicted scaling of the shear stresses $\sigma_{21} \sim \Omega^{2 / 3}$ for the hydrodynamic flow regime for Newtonian fluids are experimentally verified with PDMS melts of different viscosities. The analysis is extended to power-law fluids, and the consistency of theoretical predictions and experimental observation was shown for a strongly shear-thinning PIB in pristane solution. Finally, it is shown that for a known misalignment $\alpha$ of the triborheometrical configuration the setup can be used to determine the high shear viscosity of an unknown sample, in the current setup up to shear rates of $10^{5} \mathrm{~s}^{-1}$.

Keywords: tribology, triborheometer, hydrodynamic lubrication, high shear rheology, high shear rates, power-law fluid, Reynolds' equation

\section{Introduction}

Tribology and hydrodynamic lubrication have traditionally been considered to be a subject apart from classical bulk rheology. The main reason for this separation is that although fluid rheology contributes in each field to the observed flow and friction phenomena, the experimental approach and the resulting terminology 
differ substantially and prohibit a direct translation of the results. In particular the lack of well-defined viscometric kinematics for tribological experiments, as well as the difficulty in achieving sufficiently-precise fixture alignment in regular rheometry on the micro scale has hindered the merging of the results from the two fields. This is in particular disappointing, since the rheology of thin films yields information that localized microrheological techniques cannot provide. The dynamics of particulate probes (Gittes et al. 1997; Mason et al. 1997; Crocker et al. 2000; Levine and Lubensky 2000; Bishop et al. 2004), diffusing wave spectroscopy (Pine et al. 1988; Popescu et al. 2002) or AFM techniques (Montfort et al. 1991; Boskovic et al. 2002) that have primarily been related with rheology on the (sub)micrometer scale (Waigh 2005) test the sample only localized around the probe. However, measuring the material properties of a fluid film with a micro- to nanometer thickness allows one to access the bulk property response of a confined sample. This enables one to identify the effects of confinement on the fluid rheology as a single dimension of the bulk flow is decreased over a range of length scales until the 'tribological' interaction of the surfaces via the confined sample microstructure and the lubrication properties of the fluid dominate the response of the system.

From an experimental point of view, tribology and bulk shear rheology are very similar. Both techniques determine a shear force that is transferred from one surface to another as a function of the sliding velocity. Tribology describes this transmission of a force between two shearing interfaces via the direct interaction of these surfaces (established by a normal load that forces the surfaces into contact), moderated via a lubricating liquid. Shear rheology on the other hand describes the transmission of forces between two shearing interfaces solely via the fluid, carefully avoiding the influence of any direct interaction of the surfaces or any kinematic discontinuities at the interfaces such as slip. However, gap distances of (sub)micrometers between the two shearing surfaces can be investigated with both, tribology and shear rheology. In tribological experiments such micrometer gap distances are reached when hydrodynamic lubrication pushes the surfaces apart. A dominant stress response of the fluid at these high velocities allows then in principle to access the viscous properties of the fluid if the geometrical parameter of the surface and the gap between them is known. 
However, in order to allow for a precise alignment, tribological devices often use point contacts and thus curved surfaces that do not provide a constant gap over the shearing area. Calculation of absolute rheological parameters is therefore often not possible.

On the other hand shear rheological setups with defined gap profiles encounter difficulties with alignment and maintaining the gap for such small shearing surface separations. Experimental approaches that push the gap spacing between the shearing surfaces down to micrometer range include the microgap rheometer of Granick and co-workers that is capable of operating at 'mesoscale' gaps of 3 $500 \mu \mathrm{m}$ (Dhinojwala and Granick 1997; Mukhopadhyay and Granick 2001) and which can perform small amplitude oscillatory shear experiments (Soga et al. 1998). Several designs of piezoelectric oscillators allow probing the linear viscoelastic regime of liquids at gap settings between 10 and $100 \mu \mathrm{m}$ (Cagnon and Durand 1980; Fritz et al. 2003; Crassous et al. 2005; Vadillo et al. 2012). FMR type sliding plate rheometers (Clasen and McKinley 2004; Clasen et al. 2006; Kojic et al. 2006; Ewoldt et al. 2007; Erni et al. 2011; Clasen 2012) are capable of approaching even submicrometer gap separations (Clasen et al. 2010). Although the FMR rheometers are capable of determining normal force in the confined fluid (Baik et al. 2011), none of these shear rheometers is capable of applying a normal force and to perform a tribological experiment. Recent experimental approaches also utilize commercial rotary rheometers and their precise position, velocity and torque control in order to access shearing gaps below $100 \mu \mathrm{m}$ as described by J.R. Stokes and co-workers (Davies and Stokes 2008). As they have demonstrated, a careful correction of results obtained with a plate-plate geometry, taking into account inertia (Macosko 1994) and gap errors due to non-parallelism (Connelly and Greener 1985; Kramer et al. 1987) allows to superpose rheometrical data of a simple fluid onto the bulk master curve even for gaps down to $20 \mu \mathrm{m}$. However, the required alignment of the shearing surfaces when setting a (variable) gap below this limit and on the order of (sub)micrometers is beyond the capabilities of commercial rheometers and provide a major challenge for custom setups.

Modern commercial rotational rheometer can in principle also be used to perform tribological measurements as they are capable of applying defined rotational velocities as well as normal loads simultaneously to a sample. Furthermore, the 
highly sensitive force transducers are capable of determine even low frictional forces, and the capability of stress controlled rheometers to apply a controlled force rather than a velocity have the potential to precisely access stick-slip phenomena in tribological experiments. Another advantage of a 'triborheometrical' experiment would be the use of defined shearing areas and flow profiles and thus the possibility to calculate actual stresses acting on the fluid and surfaces. Regular tribological geometries as for example the ball-on-threeplate fixture are already commercially available (Bombard and de Vicente ; Heyer and Lauger 2009). However, rheological geometries as parallel plates for example are more difficult to operate in a tribological mode, again due to alignment issues when contacting the flat surfaces. Gong and coworkers introduced the possibility to operate the parallel plate geometry of rotational rheometer with soft surface materials in a tribological mode in order to determine the tribological response of hydrogel surfaces (Gong et al. 1999; Kagata et al. 2002; Kagata et al. 2003). By applying a known normal force they brought the flexible shearing surfaces of gel blocks mounted onto the parallel plates into direct contact. In order to achieve a parallel alignment of the contacting surfaces they exploit the elasticity of the hydrogel surfaces. With the application of a normal load these surfaces deform and level out any misalignment of the plate-plate geometry (Gong et al. 1999). Recently Kavehpour et al. proposed an experimental protocol to operate also the stiff surfaces of a parallel plate setup of a commercial rheometer in a tribological mode (Kavehpour and McKinley 2004). By applying a known normal force they confined the fluid between the parallel plates and brought the shearing surfaces into direct contact. Crucial for operating a conventional rheometer in such a 'triborheometry' mode with an applied normal force is the alignment of the stiff plates and the determination of the effectively remaining alignment error. Kavehpour et al. developed an experimental technique to align the shearing surfaces directly parallel towards each other, thus maintaining only the alignment error of the plates towards the rotational axis. This practical approach has proven to give reproducible results.

However, the effect of the remaining misalignment, in particular on the higher velocity regime is still under investigation. Any non-parallelism or non-flatness of the plates leads eventually to a hydrodynamic lubrication flow between the shearing surfaces. For a known misalignment and with the defined geometrical 
parameters of the plates the additional normal forces arising from the lubrication flow could be quantitatively calculated from solving Reynolds' equation. A numerical solution of this problem has recently been demonstrated by de Vicente et al. (Andablo-Reyes et al. 2010; Andablo-Reyes et al. 2011a; Andablo-Reyes et al. 2011b) for the lubrication flow between misaligned parallel plates in a rotational rheometer.

The aim of the current paper is to introduce an analytical solution of Reynolds' equation for the misaligned plate problem. With this we will explain the scaling of sliding friction with angular velocity that is observed in triborheological experiments with the annulus-on-plate geometry. Furthermore we will demonstrate that this analytical solution enables one to directly obtain a high shear rate flow curve from the sliding friction in the hydrodynamic lubrication regime. The paper is structured as follows: The Experimental section introduces the working principle of the triborheometrical measurements with a commercial rheometer, as well as the relevant sample fluid properties. The Results and Discussion section first shows the results of triborheometrical experiments in form of Stribeck curves. This is followed by an analytical solution of Reynolds' equation for the misaligned annulus-on-plate geometry in order to explain a power-law scaling in the hydrodynamic lubrication regime of the Stribeck curves. In the next section two different types of hydrodynamic lubrication are identified in the experiments and explained with the lubrication solution. In the following section the lubrication solution is expanded to power law-fluids as an example of a shear rate dependent viscosity model. Finally it is shown that the lubrication solution enables one to calculate viscosities and shear rates for an unknown fluid directly from the measured sliding friction and angular velocity using the known misalignment angle of the geometry.

\section{Experimental}

\section{Test fluids}

The investigated test fluids were polydimethyl siloxane (PDMS) melts from Gelest Inc. (Tullytown, PA) with nominal viscosities of $1000 \mathrm{cSt}, 5000 \mathrm{cSt}$ and 
$10000 \mathrm{cSt}$. A lower viscosity PDMS with a nominal viscosity of $350 \mathrm{mPas}$ (Rhodorsil silicone oil 47V350) was supplied by VWR Int. SAS (Briare, France). As a shear thinning sample a solution of a high molecular weight polyisobutylene (PIB, Sigma-Aldrich) dissolved in 2,6,10,14-tetramethylpentadecane (pristane, Sigma-Aldrich) with a PIB mass fraction of 0.114 was prepared (for details see (Vananroye et al. 2010)). This fluid has similar components as the NIST Standard Reference Material SRM 2490 that shows a characteristic viscoelastic behavior similar to that of polymeric melts with the advantage of being liquid at room temperature (Schultheisz and Leigh 2002).

\section{Bulk rheological and high shear characterization of the test fluids}

The viscosity as well as the first normal stress differences $N_{l}$ as a function of the shear rate $\&$ were determined with an AR-G2 rotational rheometer (TA Instruments, Newcastle, DE) and a cone and plate fixture $\left(\varnothing=4 \mathrm{~cm}, 1^{\circ}\right.$ cone angle), the results of these bulk rheological measurements are presented in Fig. 1. In addition to this, the high shear response of selected samples determined with an FMR is shown (data taken from (Clasen et al. 2010)). These results that coincide with the bulk rheological measurements extend the shear rate ranges up to 20000 $\mathrm{s}^{-1}$ and thus to shear rates as they occur in the hydrodynamic lubrication regime of the triborheometrical experiment. At these high shear rates the flow curves of the higher viscosity PDMS samples indicate the onset of a shear thinning regime and the appearance of a first normal stress difference. The PDMS melts are thus not Newtonian fluids. Still, the decrease in viscosity over the relevant shear rate range for the following discussion is small, and we treat and call these samples 'quasiNewtonian' fluids. For calculations requiring the viscosity we use, however, the exact viscosities determined in Fig. 1. For the high shear rates also the expected $N_{1}$ evolution obtained from the flow curves via the White-Metzner model is given as described in (Clasen et al. 2010).

As shown in detail in Appendix A the investigated fluids are not affected by viscous heating, pressure or inertial effects in the triborheometrical experiments.

Figure 1 


\section{The plate-on-annulus geometry for triborheometrical experiments}

Tribological measurements of the different samples were conducted using a rotational triborheometer setup as described by Kavehpour and McKinley (Kavehpour and McKinley 2004). This triborheometer utilizes a regular rotational rheometer (for the present investigation an AR2000 and an AR-G2 rotational rheometer (both TA Instruments, Newcastle, DE)) with a parallel plate geometry as the upper shearing surface to apply a constant normal load and angular velocity to a sample and to determine the resulting torque. As the lower shearing surface an annular fixture was utilized as schematically shown in Fig. 2. Three different plate/annulus combinations were used:

- An annulus with an inner radius of $R_{1}=2.25 \mathrm{~cm}$ and an outer radius of $R_{2}$ $=2.54 \mathrm{~cm}$ made from $\lambda / 20$ optical flat glass For the upper shearing surface a regular parallel plate fixture (stainless steel) of the AR2000 with a diameter of $R=6 \mathrm{~cm}$ was used.

- A second annulus made from stainless steel with $R_{1}=1.47 \mathrm{~cm}$ and $R_{2}=$ $0.76 \mathrm{~cm}$. The upper shearing surface was in this case a $4 \mathrm{~cm}$ diameter steel plate fixture of the AR2000.

- A third annulus was made from aluminum $\left(R_{1}=2.037 \mathrm{~cm}\right.$ and $R_{2}=2.262$ $\mathrm{cm}$ ) and used against a $6 \mathrm{~cm}$ diameter steel plate fixture of the AR-G2.

\section{Figure 2}

Following Kavehpour and McKinley the shearing surface of the annulus is aligned to the upper surface by placing a small amount of wax between the ring and the peltier element of the rheometer on which the annulus is centered. The wax is then heated via the peltier element to its melting temperature and the upper surface connected to the rheometer is lowered to press the annulus into the wax. Cooling the wax below its melting point arrests the annulus surface in its now parallel position to the upper surface. The annulus is then further fixed in this position to the peltier with fast curing epoxy glue. The effective misalignment $\alpha$ 
between the two plates is thus reduced to the non-perpendicularity of the top plate to the rotational axis, and to any non-flatness of the top or bottom shearing surface.

For the triborheometrical measurement a sample is placed between the annulus and the top shearing surface and the top shearing surface is pressed with a desired normal load $F_{N}$ onto the sample (with a tolerance of $\pm 0.3 \mathrm{~N}$ ). A slight overfilling ensures a filled gap even for a widening of the gap as discussed later. The upper plate is then rotated with a constant angular velocity $\Omega$ and the torque $T$ is recorded. For the current investigation each data point is determined over a time period of at least $1200 \mathrm{~s}$. The reported data are steady state data for which the averaged torque over a full rotation of the upper plate stayed within $\pm 3 \mathrm{mNm}$ for 3 consecutive rotations. A detailed description of the experimental conditions and raw data treatment can be found in Appendix A. As shown there the readout of the absolute gap from the rheometer is not reliable due to thermal expansion within the rheometer. However, the gap variation readout over a full rotation of the fixture at low velocities allows an estimation of the remaining misalignment angle of the plates.

\section{Results and Discussion}

\section{Triborheometrical experiments and Stribeck curves}

The results of triborheometrical measurements with the annulus fixtures are shown in Fig. 3 for different PDMS test fluid, normal loads, and shearing surface combinations.

\section{Figure 3}

These so called Stribeck curves give the dimensionless sliding friction $\mu$ as a function of the Gumbel number $G u$. As commonly reported for tribology measurements, $\mu$ is the ratio of the measured shear force $F_{S}$ and the applied normal load $F_{N}$

$$
\mu=\frac{F_{S}}{F_{N}}
$$


For the small width of the annulus geometry in the current setup this shear force is related to the torque $T$

$$
F_{S}=\frac{2 \bar{R}}{R_{1}^{2}+R_{2}^{2}} T
$$

where $\bar{R}$ is the average radius of the annulus

$$
\bar{R}=\frac{\left(R_{1}+R_{2}\right)}{2} .
$$

The dimensionless Gumbel number $G u$ is a measure for the relative velocity of the sliding surfaces (Kavehpour and McKinley 2004)

$$
G u=\frac{\eta \Omega A}{F_{N}}
$$

where $\eta$ is the nominal viscosity of the fluid, $\Omega$ is the angular velocity of the rotating shearing surface and $F_{N} / A$ is the average normal stress acting on the shearing surface area $A$ of the annulus

$$
A=\pi\left(R_{2}^{2}-R_{1}^{2}\right)
$$

The Stribeck curves in Fig. 3 show in general three distinct regimes:

- At low Gumbel numbers the sliding friction between the shearing surfaces is dominant. In this 'boundary lubrication' regime, the friction coefficient $\mu$ is independent of the relative velocity between the shearing surfaces. This is counterintuitive for the rheologist for whom rate independent stresses are normally encountered only for yielding processes. However, following Coulomb's friction laws $\mu$ is in this regime constant for a surface material/fluid type combination, and independent of the fluid viscosity and the applied normal force. - At high velocities in the 'hydrodynamic lubrication' regime the stress response is dominated by the fluid rheology. At these velocities the stresses that arise in the sheared fluid itself are larger than any direct frictional stress between the surfaces. - At intermediate velocities one can observe a transitional regime, denominated 'mixed lubrication', at which both hydrodynamic and boundary lubrication are present. This regime is very sensitive to a range of parameters from surface roughness to the internal microstructure of the fluid and therefore often most interesting for the investigation of the lubricating properties of a certain surface/liquid combination. In general, the mixed lubrication regime exhibits 
smaller stress values then the boundary lubrication and a characteristic minimum is observed. For the rotating plate-on-annulus configuration used in Fig. 3 the mixed lubrication regime is generally not very distinct. Still, it can be noted that the magnitude of the minimum is following the roughness of the employed surfaces: For the glass on steel surface combination with a very small surface roughness of the glass the characteristic minimum is not observed at all.

An advantage of the plate-on-annulus configuration is the possibility to convert the tribological dimensionless numbers of the Stribeck curves into rheological nomenclature. The narrow width annulus can be treated in good approximation as an infinitely long sliding plate configuration with the free interfaces exposed to ambient pressure (it is ensured in the experiments that the inside of the annulus is in contact with the surrounding atmosphere via a small opening at the bottom of the annulus, also to avoid compression of trapped air during sample loading). With this it is possible to calculate the shear and normal stresses from the forces (Xu et al. 2007) via

$$
\begin{aligned}
& \sigma_{21}=F_{S} / A \\
& N_{1}=F_{N} / A
\end{aligned}
$$

and thus to correlate the friction coefficient to the shear stress

$$
\mu=\sigma_{21} \frac{A}{F_{N}}
$$

To convert the Gumbel number $G u$ into a shear rate $\dot{\gamma}$, the relatively small ratio of annulus width to inner radius of the annulus, $\left(R_{2}-R_{1}\right) / R_{1}$ allows the assumption of only a small variation of shear rate across the annulus width. In the following we assume therefore in good approximation a constant average values of the shear rate $\overline{\dot{\gamma}}$ obtained at the average radius $\bar{R}$ of eq. (3) (and denote this in the following simply as $\dot{\gamma}$ ).

The shear rates $\dot{\gamma}$ relates to the angular frequency $\Omega$ via

$$
\dot{\gamma}=\frac{\Omega \bar{R}}{h_{e f f}}
$$


where $h_{\text {eff }}$ is the effective gap that the sheared sample experiences over a full rotation of the shearing surface. If this effective gap $h_{\text {eff }}$ is known or can be measured it is possible to convert the Gumbel number into a shear rate

$$
G u=\not \& \frac{\eta A h_{\text {eff }}}{F_{N} \bar{R}}
$$

The rotational rheometers used in the current triborheometer setup are in principle able to determine the absolute position of the upper plate and therefore the absolute gap. However, it is shown in Appendix A that an uncontrolled thermal expansion of the rheometer shaft is prohibiting a direct determination of the absolute value of $h$.

As pointed out by Kavehpour and McKinley (Kavehpour and McKinley 2004) for a fluid with a known dependence of the viscosity on the shear rate

$$
\eta(\dot{\gamma})=\frac{\sigma_{21}(\dot{\gamma})}{\dot{\gamma}},
$$

it is possible to correlate a Gumbel number $G u$ with a shear rate $\dot{\gamma}$ and to calculate then the effective gap from eq. (9). This correlation is done by matching the shear stress level $\sigma_{21}(G u)$ of the hydrodynamic lubrication regime with the shear stress $\sigma_{21}=\eta(\dot{\gamma}) \dot{\gamma}$ determined with conventional bulk rheometry. This method as originally introduced by Conelly and Greener (Connelly and Greener 1985 ) is demonstrated in Fig. 3: the dashed line gives $\mu$ as a function of Gumbel numbers that have been calculated with eq. (9) from the bulk viscosities and shear rates of a curve of Fig. 1. Fitting this curve to match the onset of the hydrodynamic lubrication regime in Fig. 3 yields a value for the effective gap $h_{\text {eff }}$ of $2 \mu \mathrm{m}$. Although it is debatable if this effective gap is really representative for the onset of the hydrodynamic lubrication regime, it becomes in particular apparent from Fig. 3 that using such a constant $h_{\text {eff }}$ does not allow matching the hydrodynamic lubrication regime over the full observation range. The observed slope of the hydrodynamic lubrication regime in Fig. 3 shows a scaling of the shear force with the velocity with a power of $2 / 3$ and is therefore less than the indicated slope of 1 obtained for a constant effective gap with eq. (9). Such a scaling exponent of less than unity is also present in other published experimental results (see for example Fig. 4 in (Kavehpour and McKinley 2004)). 
De Vicente et al. have observed a similar behavior in numerical simulations of a misaligned parallel plate configuration (Andablo-Reyes et al. 2010; AndabloReyes et al. 2011a; Andablo-Reyes et al. 2011b) and attributed the lower slope to a widening of the gap and a change of $h_{\text {eff }}$ due to the lubrication flow between misaligned parallel plates in a rheometer. A similar explanation had also been proposed by Clasen et al. (Clasen et al. 2007) for the plate-on-annulus configuration. Furthermore, a similar scaling of the hydrodynamic lubrication regime with a power less then unity is discussed in literature for elastohydrodynamic lubrication, also in context with a non-constant gap $h_{\text {eff }}$ (de Vicente et al. 2005; de Vicente et al. 2006), however in this case due to a deformation of the elastic surface.

To test this hypothesis of a widening gap in the hydrodynamic lubrication regime as an explanation for the observed $2 / 3$ scaling, we seek in the following an analytical solution for the lubrication forces in the plate-on-annulus configuration.

\section{Analytical solution of Reynolds' equation for hydrodynamic lubrication}

The deviation of perpendicularity of the upper shearing surfaces to the rotation axis is unavoidable in the manufacturing process of the rheometer geometries. The entrainment of liquid during the relative rotation of a not perfectly parallel plate with a tilt angle $\alpha$ to the annulus (as schematically depicted in Fig. 2) will cause a lubrication pressure that forces the plate and annulus apart. This pressure can be calculated from the steady state Reynolds' equation, which takes in two dimensions and without a squeeze term contribution the form (Williams 2005)

$$
\frac{\partial}{\partial(\theta \bar{R})}\left\{h^{3} \frac{\partial p}{\partial(\theta \bar{R})}\right\}+\frac{\partial}{\partial r}\left\{h^{3} \frac{\partial p}{\partial r}\right\}=6 \eta U \frac{d h}{d(\theta \bar{R})}
$$

where $\theta$ denotes the rotation angle and $U$ the relative velocity difference between the surfaces.

We assume that the pressure gradient in radial direction, $\partial p / \partial r$, is much larger than the gradient along the converging gap, $\partial p / \partial(\theta \bar{R})$, an assumption that is justified for a narrow width $\left(R_{2}-R_{1}\right)$ of the shearing surface in comparison to the 
length $\pi \bar{R}$. Then with a negligible gap change in radial direction, $\partial h / \partial r$, equation (11) reduces to

$$
h^{3} \frac{d^{2} p}{d r^{2}}=6 \eta U \frac{d h}{d(\theta \bar{R})}
$$

similar to the Ocvirk solution for an infinitely small pad bearing.

Integration along $r$ with the boundary conditions that the pressure drops to zero at $R_{1}$ and $R_{2}$ gives then the pressure

$$
p=\frac{3 \eta U}{h^{3}} \frac{d h}{d(\theta \bar{R})}\left\{(r-\bar{R})^{2}-\frac{\left(R_{2}-R_{1}\right)^{2}}{4}\right\} \text {. }
$$

The lifting force can be determined by a half-Sommerfeld solution (assuming that only areas with a positive $d h / d(\theta \bar{R})$ contribute to the lifting force) by integrating equation (13) along $\theta \bar{R}$ from 0 to $\pi \bar{R}$ :

$$
F_{N}=\int_{R_{1}}^{R_{2}} \int_{0}^{(\pi \bar{R})} \frac{3 \eta U}{h^{3}} \frac{d h}{d(\theta \bar{R})}\left\{(r-\bar{R})^{2}-\frac{\left(R_{2}-R_{1}\right)^{2}}{4}\right\} d(\theta \bar{R}) d r
$$

Separation of the integrals and applying the boundary conditions $h=h_{1}$ at $\theta=0$ and $h=h_{2}$ at $\theta=\pi$ gives

$$
F_{N}=\int_{R_{1}}^{R_{2}}(r-\bar{R})^{2}-\frac{\left(R_{2}-R_{1}\right)^{2}}{4} d r \int_{h_{1}}^{h_{2}} \frac{3 \eta U}{h^{3}} d h
$$

Solving the integrals results then in the lifting force

$$
F_{N}=\frac{\eta U}{4}\left(R_{2}-R_{1}\right)^{3}\left(\frac{1}{h_{1}^{2}}-\frac{1}{h_{2}^{2}}\right)
$$

Interestingly this solution does not directly depend on the tilt angle $\alpha$ of the geometry, but just on the height difference between $h_{1}$ and $h_{2}$.

With the surface area of the annulus $A$ (eq. (5)) and a velocity $U \cong \Omega \bar{R}$, we can rewrite equation (16) to obtain a Gumbel number

$$
G u=\frac{\eta \Omega A}{F_{N}}=\frac{8 \pi}{\left(R_{2}-R_{1}\right)^{2}}\left(\frac{1}{h_{1}^{2}}-\frac{1}{h_{2}^{2}}\right)^{-1} .
$$


For convenience we combine the geometrical parameters into a single constant

$$
C=\frac{2 \pi \bar{R}^{2}}{\left(R_{2}-R_{1}\right)^{2} \sin \alpha}=\frac{8 \pi^{3} \bar{R}^{4}}{A^{2} \sin \alpha} \text {. }
$$

With this and using the relation $h_{2}-h_{1}=2 \bar{R} \sin \alpha$ one obtains a relation between the Gumbel number and the gap $h_{1}$

$$
G u=4 C \sin ^{3} \alpha\left(\frac{1}{\left(\frac{h_{1}}{R}\right)^{2} \sin \alpha}-\frac{1}{\left(\frac{h_{1}}{R \sin \alpha}+2\right)^{2}}\right)^{-1}
$$

The dependency of the gap $h_{1}$ on the Gumbel number $G u$ is plotted in a recursive way in Fig. 4 for several values of the tilt angle $\alpha$.

\section{Figure 4}

The results in Fig. 4 predict not only an increasing gap as a function of a rising velocity, but also two regimes with different scaling of the reduced gap with the Gumbel number can be observed. Obviously for $h_{1}=\bar{R} \sin \alpha$ eq. (19) approaches

$$
\lim _{\left(\frac{h_{1}}{\bar{R} \sin \alpha}\right) \rightarrow 0} h_{1}=\frac{\left(R_{2}-R_{1}\right)}{4 \sqrt{\pi}} G u^{\frac{1}{2}}
$$

For gaps larger than the tilt error as expected for the hydrodynamic lubrication regime of the triborheometer, eq. (19) approaches the limiting relation

$$
\lim _{\left(\frac{h_{1}}{\bar{R} \sin \alpha}\right) \rightarrow \infty} h_{1}=\frac{\bar{R}}{C^{\frac{1}{3}}} G u^{\frac{1}{3}}
$$




\section{Comparison of the lubrication solution to experimental results}

We are now able to compare the theoretically predicted dependence of the gap on the Gumbel of eqs. (20) and (21) to experimental results. As laid out in Appendix A it is not possible to use the gap readout of the rotational rheometer due to thermal expansion within the rheometer. However, for an experimentally determined or known dependence of the viscosity on the shear rate and thus (via eq. (8)) on the stress, $\eta\left(\sigma_{21}\right)$, it is possible to directly determine with eqs. (8) and (10) the effective gap for a measured torque or shear stress $\sigma_{21}$ :

$$
h_{\text {eff }}=\Omega \bar{R} \eta\left(\sigma_{21}\right) / \sigma_{21}
$$

Since the measured stress $\sigma_{21}$ is directly related to an angular velocity $\Omega$, it is possible to report this experimental effective gap $h_{\text {eff }}$ as a function of $\Omega$ (or with eq. (4) as a function of the Gumbel number $G u$ ). This is shown for example in Fig. 5 that gives the Stribeck curve for a PDMS melt at a normal load of $5 \mathrm{~N}$. From this sliding friction $\mu$ and the separately determined viscosity $\eta$ (from Fig. 1) the corresponding effective gaps $h_{\text {eff }}$ have been calculated with eq. (22) and are also given in Fig. 5.

\section{Figure 5}

This effective gap $h_{\text {eff }}$ in the hydrodynamic lubrication regime increases with the Gumbel number and indeed with a power law dependence on the Gumbel number of $1 / 3$ as predicted by eq. (21). The dashed line in Fig. 5 is a fit of the experimental gap data with the theoretical predictions of eq. (21), using the tilt angle $\alpha$ as the only fitting parameter. The resulting angle of $\alpha=3.8 \times 10^{-4} \mathrm{rad}$ is of the same order of magnitude as the separately determined misalignment of the top plate of $\sim 1.8 \times 10^{-4} \mathrm{rad}$ (Appendix A).

At high Gumbel numbers in Fig. 5 (above $G u=0.001$ ) the effective gap $h_{\text {eff }}$ shows a strong increase above the theoretical predictions of eq. (21). This goes along with a leveling of the sliding friction into a plateau value independent of the Gumbel number. The reason for this behavior becomes obvious when comparing 
the applied normal stress in Fig. 5 to the normal stresses arising from the fluid itself. The relation between $\dot{\gamma}$ and the angular velocity $\Omega$ that enters the Gumbel number is obtain from inserting eqs. (21) and (4) into (8):

$$
\dot{\gamma}=\Omega^{\frac{2}{3}}\left(\frac{C F_{N}}{A \eta}\right)^{\frac{1}{3}}
$$

At a Gumbel number of $\mathrm{Gu}=0.001$ (or a shear rate of $\dot{\gamma}=3500 \mathrm{~s}^{-1}$ ) the first normal stress difference $N_{1}$ of the fluid reaches the applied $N_{1}$. Any increase of the angular velocity beyond this point leads to an additional widening of the gap, so that the shear rate in the gap and therefore the fluids $N_{1}$ will stay constant and just balance the applied $N_{1}$. As a consequence, also the shear stress and thus the hydrodynamic sliding friction will stay constant with increasing Gumbel number. The observed plateau for the sliding friction represents therefore the upper limit of the triborheometrical experiment of this fluid and this applied normal force. For example in Fig. 3 using the same shearing surface combination but a PDMS with a lower viscosity of $\eta=0.35$ Pas, it is possible to follow the sliding friction to higher Gumbel numbers, although the applied $N_{1}$ is lower. This is because the $N_{1}$ of the fluid is much lower (not measurable with the rotational rheometer in Fig. 1) and not reaching the value of the applied $N_{1}$ over the range of applied Gumbel numbers.

\section{Two different types of hydrodynamic lubrication}

An increase of the gap with increasing velocity for hydrodynamic lubrication is not a general rule. As for example shown in (Kavehpour and McKinley 2004) a constant effective gap could be assumed in the hydrodynamic regime for several of their samples. Also for our simple test fluids it was possible to select a combination of shearing surface material (stainless steel annulus against a steel plate) to observe a constant effective gap $h_{\text {eff }}$ even in the hydrodynamic lubrication regime. For this surface combination in Fig. 6 the friction factors and angular velocities are converted into shear stresses and rates with eqs. (7) and (8). As it can be seen, it was possible to shift the lower part of the hydrodynamic lubrication regime onto the bulk flow curve, using constant effective gaps as indicated in the figure legend. 
Furthermore, with increasing rate it is possible in Fig. 6 to observe a sudden transition to a different scaling regime (indicated as 'type II').

\section{Figure 6}

This transition to a different scaling regime is even more obvious in Fig. 7 when plotting the data of Fig. 6 again as Stribeck curves. Here are clearly two different mechanisms of hydrodynamic lubrication observed:

For type I hydrodynamic lubrication above a critical rotational velocity the stress response of the sheared fluid (between the not perfectly parallel plates) becomes larger than the resistance caused by the frictional interaction at the contact point of the plates. However, the lubrication force is at these velocities not high enough to overcome the applied normal force and the effective gap stays constant at a minimum value $h_{\text {eff }, 0}$. The observed shear stress $\sigma_{21}=\eta \dot{\gamma}$ depends then with the shear rate formulation of equation (8) linearly on the angular velocity:

$$
\sigma_{21}=\frac{\eta \bar{R}}{h_{e f f, 0}} \Omega
$$

as also indicated in Fig. 6. A translation to tribological dimensionless numbers gives

$$
\mu=\frac{\bar{R}}{h_{e f f, 0}} G u .
$$

and therefore the slope of 1 observed at lower Gumbel numbers in Fig. 7 In case that a type I lubrication with $\sigma_{21} \sim \Omega$ is actually observed the constant effective gap $h_{\text {eff }, 0}$ can simply be determined by shifting the data onto the bulk flow curve as done in Fig. 6.

The possibility to observe a type I regime is, however, closely connected to the type of geometrie used: the parallel surfaces plate-on-annulus geometry lead for a small misalignment to relatively large non-contacting shearing areas with a small separation. This leads to high enough shear rates over larger areas so that the fluid can provide sufficiently large shear stresses even when the surfaces are still in a direct frictional point contact. Furthermore, increasing the shearing surface area 
by increasing the width $\left(R_{2}-R_{1}\right)$ of the annulus (as done for the steel on steel geometry in Fig. 6) is going to promote the appearance of a type I regime.

However, it should be noted that leaving the narrow width limit for $\left(R_{2}-R_{1}\right)$ will eventually lead to a deviation from the quantitative lubrication solution of eq. (19).

Similar observations of such a constant gap lubrication have for example been reported by Gong and co-workers for the case of osmotic induced hydrated lubrication (Oogaki et al. 2009). In their case the average gap between the shearing surfaces is solely determined by the osmotic repulsive pressure of the electric double layers and stays constant independent of the sliding velocity. At low sliding velocities the frictional interactions of the surface asperities are still dominating. However, at high enough sliding velocities the viscous stresses of the fluid in the constant gap dominates, leading to a linear dependence of the stress on the velocity as given for a type I regime by eq. (24) (Oogaki et al. 2009).

For type II hydrodynamic lubrication the rotational velocity and lubrication forces are high enough to provide sufficient lifting normal force against the applied normal force. The resulting plate separation is large enough to let the stress caused by friction of the boundary lubrication become smaller then the hydrodynamic stresses. Only in case that at the onset of the lifting $h_{1}=\bar{R} \sin \alpha$ (and the slowly increasing gap follows ideally the Gumbel number with equation (20)) the gap will still be dominated by the constant $h_{e f f, 0}$ and $\sigma_{21} \sim \Omega$ still holds.

\section{Figure 7}

However, as soon as $h_{1} ? h_{e f f, 0}$ the increase of the effective gap with the angular velocity will become observable in the shear stress evolution. In this type II lubrication regime one can assume (for $h_{1} \gg h_{\text {eff }, 0}$ ) that $h_{1}(\Omega) \approx h_{\text {eff }}(\Omega)$. One can then insert the theoretical gap height $h_{1}$ of equation (21) into eq. (24) or (25) to replace the varying effective gap $h_{\text {eff }}$ and to obtain the stress evolution with the angular velocity: 


$$
\sigma_{21}=\left(\frac{\eta^{2} F_{N} C}{A}\right)^{\frac{1}{3}} \Omega^{\frac{2}{3}},
$$

or the sliding friction evolution with the Gumbel number

$$
\mu=C^{\frac{1}{3}} G u^{\frac{2}{3}}
$$

It is this scaling of $\mu$ with a power of $2 / 3$ with $G u$ that is clearly observed in Fig. 7 at higher $G u$, and that is the generally observed behavior in Fig. 3 and also expected and clearly observed in Fig. 5.

As a not on the side: by inserting eq. (21) into eq. (27) we can obtain for the type II regime a direct relation between the sliding friction and the gap, which is independent of the viscosity of the sample. For this relation that has so far only been numerically approached by de Vicente et al. for misaligned parallel plates geometries (Andablo-Reyes et al. 2010) we obtain now a quantitative description:

$$
h_{1}=\frac{\bar{R}}{C^{\frac{1}{2}}} \mu^{\frac{1}{2}} .
$$

When comparing the Stribeck curves of Figs. 5 and 7 it is obvious that a type I regime is not always observable. In case that the stress level of the boundary and mixed lubrication regime are already larger than any stress level in the type I hydrodynamic lubrication regime one will observe a direct transition to the $\eta$ : $G u^{2 / 3}$ scaling and a type II hydrodynamic lubrication.

A critical Gumbel number $G u_{c r}$ up to which a type I lubrication might be observable can be obtained by matching the sliding frictions of equations (25) and (27). The transition between the type I and type II regime happens then at

$$
G u_{c r}=C\left(\frac{h_{e f f, 0}}{\bar{R}}\right)^{\frac{1}{3}} \text {. }
$$

This critical Gumbel number depends on the minimum effective gap $h_{\text {eff }, 0}$ of regime type I and the tilt angle $\alpha$. This critical Gumbel number relates also to a critical friction coefficient

$$
\mu_{c r}=C\left(\frac{h_{e f f, 0}}{\bar{R}}\right)^{2} .
$$


A closer look at these critical numbers reveals why the transition from regime type I to type II is observed for the Stribeck curves in the steel on steel geometry in Fig. 7 but not for the aluminum/steel geometry in Fig. 5. For both surface combinations one obtains from the geometrical parameters and observed angles in Fig. 5 and 7 roughly the same $C / \bar{R}^{2}$ in eq. (30). Then for the same $h_{\text {eff }, 0}$ (estimated from the fits in Fig. 7 as $h_{\text {eff }, 0} \approx 6 \mu \mathrm{m}$ ) the level of critical sliding friction will be of the same order of magnitude of $\mu_{c r} \sim 0.2$. However, for the experiments of the aluminum on steel combination the observed level of $\mu$ in the boundary lubrication regime in Fig. 5 (and 3) is already at this critical value of 0.2 . Any hydrodynamic lubrication will therefore be above $\mu_{c r}$ and only type II is observed. On the other hand for the steel/steel combination the boundary lubrication in Fig. 7 is an order of magnitude lower then $\mu_{c r} \sim 0.2$, and the observation of the type I is therefore possible.

One thing to note is that while it is easy to determine the minimum effective gap $h_{e f f, 0}$ when a type I regime is actually observed (as demonstrated in Fig. 6), an $a$ priori estimation of $h_{\text {eff }, 0}$ is difficult. The minimum effective gap $h_{\text {eff }, 0}$ of the type I lubrication regime is not directly related to be the gap distance change $\Delta h$ along a rotation of the annulus determined in Appendix A (where $\Delta h$ could be related to the tilt angle $\alpha$ as shown in the example of Fig. 5). Comparing in Fig. 7 the $\Delta h=$ $0.8 \mu \mathrm{m}$ obtained from tilt angle $1.8 \times 10^{-5} \mathrm{rad}$ of the Type II regime via eq. (42) to $h_{\text {eff }, 0} \sim 6 \mu \mathrm{m}$ shows an order of magnitude difference. The question that remains is what determines the minimum effective gap $h_{e f f, 0}$ in the type I regime. Although the stress response is determined by the rheology of the fluid, the distance between the plates will still be on the same order as for the boundary lubrication regime. Surface roughness could play in this case role. However, as already reported by (Kavehpour and McKinley 2004), tests conducted with the triborheometer reveal that a surface roughness variation covering a range from $\varepsilon=$ $0.7-678 \mathrm{~nm}$ did not lead to a change of the sliding friction of a synthetic oil with comparable viscosity ( $\eta=0.7$ Pas) in the boundary lubrication regime. Furthermore, the range of investigated surface roughness is still an order of magnitude below the observed minimum effective gaps $h_{\text {eff }, 0}$. Recently Clasen et al. have shown with thin film rheometry that the PDMS oils contain traces of 
deformable impurities with dimensions on the order of micrometers that can affect the fluid response already at micrometer shearing surface separations comparable to the observed $h_{e f f, 0}$ (Clasen et al. 2010). Another possible origin of $h_{e f f, 0}$ can be a non-flatness of one or both shearing surfaces. Even for surfaces that are perpendicular to the rotation axis and that have a maximum parallel alignment such a non-flatness would lead to a remaining average gap of the order of the nonflatness. It should be noted that a non-flatness would also cause an additional lubrication flow. The effective misalignment angles $\alpha$ that are obtained from fitting the type II regime in Figs. 5, 7 or 8 will therefore contain contributions from both non-parallelism and non-flatness (if present).

\section{Power-law fluids in hydrodynamic lubrication}

The above analysis assumed the viscosity of the fluid to be constant (or to have an experimentally determined dependence on the rate). One can, however, easily expand the Newtonian case to any rate dependent viscosity model. As an easy example in the following we assume a power-law fluid with a shear rate dependent viscosity

$$
\eta=K \dot{\gamma}^{n-1}
$$

where $n$ is the power-law index ranging from 0 to 1 and $K$ is a consistency index. Inserting this viscosity into equation (24) for a type I hydrodynamic lubrication yields the shear stress as a function of angular velocity:

$$
\sigma_{21}=K \dot{\gamma}^{n}=\frac{K \bar{R}^{n}}{h_{e f f, 0}^{n}} \Omega^{n}
$$

and thus a sliding friction that shows a power-law dependence on the angular velocity

$$
\mu=\frac{K A}{F_{N}} \frac{\bar{R}^{n}}{h_{e f f, 0}^{n}} \Omega^{n} .
$$

Using the power-law viscosity also in the Gumbel number and inserting eqs. (31) and (8) into eq. (4) gives 


$$
G u_{n}=\frac{K A}{F_{N}}\left(\frac{\bar{R}}{h_{e f f, 0}}\right)^{n-1} \Omega^{n} .
$$

The sliding friction and Gumbel number show the same relation $\mu=\left(\bar{R} / h_{\text {eff }, 0}\right) G u_{n}$ as for the Newtonian liquid in equations (25).

For the hydrodynamic lubrication type II regime one has to take into account that the viscosity also enters the velocity dependent gap $h_{l}$ of equation (21) and therefore also the effective shear rate. Inserting equation (21) into equation (8) (again with the assumption that $h_{\text {eff }} \cong h_{1}$ for $h_{1} \gg \bar{R} \sin \alpha$ ), using the power-law of eq. (31) for the viscosity $\eta$ and solving for $\dot{\gamma}$ gives the shear rate in the gap for a power-law fluid:

$$
\dot{\gamma}=\left(\frac{F_{N} C}{K A}\right)^{\frac{1}{n+2}} \Omega^{\frac{2}{n+2}}
$$

Inserting (35) into (31) gives then the viscosity as a function of the angular velocity

$$
\eta=\left(\frac{K^{\left(\frac{3}{n-1}\right)} F_{N} C}{A}\right)^{\frac{n-1}{n+2}} \Omega^{\frac{2 n-2}{n+2}}
$$

The shear stress in the type II regime is then obtained by inserting equation (31) and (35) into equation (10):

$$
\sigma_{21}=\left(\frac{K^{\frac{2}{n}} F_{N} C}{A}\right)^{\frac{n}{n+2}} \Omega^{\frac{2 n}{n+2}}
$$

With this the sliding friction for a power law fluid shows the following dependence on the angular velocity

$$
\mu=\left(\frac{K A}{F_{N}}\right)^{\frac{2}{n+2}} C^{\frac{n}{n+2}} \Omega^{\frac{2 n}{n+2}}
$$


Finally, using the power-law viscosity also in the Gumbel number by inserting (31) and (35) into equation (4) leads to

$$
G u_{n}=\left(\frac{K A}{F_{N}}\right)^{\frac{3}{n+2}} C^{\frac{n-1}{n+2}} \Omega^{\frac{3 n}{n+2}}
$$

It can be noted that combining equations (38) and (39) gives again the general dependence of a type II lubrication of $\mu=C^{\frac{1}{3}} G u^{\frac{2}{3}}$ of eq. (27). Furthermore it can easily be shown that both sliding friction and Gumbel number of eqs. (38) and (39) reduce for a power-law exponent of $n=1$ to the general Newtonian case of definitions of eqs. (27) and (4).

\section{Figure 8}

An experimental verification of this scaling of the sliding friction for a type II hydrodynamic lubrication of a power-law fluid can be seen in Fig. 8. Here a quasi Newtonian PDMS melt is compared to a PIB in pristane solution as a model power-law fluid. Both fluids have a similar viscosity at a shear rate of $10.000 \mathrm{~s}^{-1}$, which is representative for the order of magnitude of rates encountered in triborheometrical experiments (the flow curves are also given in the top part of Fig. 8, and the shear rate axes of the top graph are matched with the angular velocity axis in the bottom graph via eq. (35)).

While the quasi Newtonian fluid shows the expected scaling of $\mu \sim \Omega^{2 / 3}$, the slope of the power-law fluid is lower. The observed scaling of $\mu \sim \Omega^{0.29}$ is consistent with the prediction of eq. (38). From the flow curve a power-law exponent of $n-1$ $=-0.665$ can be obtained that leads in eq. (38) to an exponent for the sliding friction of $2 n /(n+2)=0.287$. this is in excellent agreement with the experimentally observed slope of 0.29 in Fig. 8. The dotted and solid lines in Fig. 8 are fits of the theoretical predictions of the power-law and the Newtonian case from eqs. (38) and (27) respectively to the experimental data, using the viscosities of the extrapolated flow curves in the top graph of Fig. 8. The only fitting parameter was in both cases the tilt angle $\alpha$ that gave for the two different fluids similar values of $\alpha=1.90 \times 10^{-4} \mathrm{rad}$ and $\alpha=1.86 \times 10^{-4} \mathrm{rad}$. 


\section{High shear rate rheometry using hydrodynamic lubrication}

From a rheological point of view, the hydrodynamic lubrication regime of the triborheometer enables one to access relatively high shear rates. Taking a closer look at the lubrication solution of the type II regime (eq. (26) reveals that the measured stress depends on the angular velocity and viscosity, but next to this only on geometrical parameters. A triborheometrical geometry with a known value of the misalignment angle $\alpha$ that enters the geometrical parameter constant $C$ of eq. (18) can therefore in principal be used as a novel type of rheometrical tool to obtain the high shear rate viscosity of an unknown sample. Solving equation (26) for the viscosity gives

$$
\eta=\frac{\sigma_{21}^{\frac{3}{2}}}{\Omega}\left(\frac{A}{C F_{N}}\right)^{\frac{1}{2}} .
$$

This high shear viscosity can thus be obtained from the measured shear stress

$\sigma_{21}(\Omega)$ at an applied angular velocity $\Omega$. Similarly, inserting eq. (40) into eq. (23) gives the shear rate $\dot{\gamma}$ for a measured $\sigma_{21}(\Omega)$ :

$$
\&=\frac{\Omega}{\sigma_{21}^{\frac{1}{2}}}\left(\frac{C F_{N}}{A}\right)^{\frac{1}{2}}
$$

It is therefore possible to obtain the viscosity as a function of the shear rate, $\eta(\dot{\gamma})$, simply from the measured stress as a function of the angular velocity, $\sigma_{21}(\Omega)$, once the angle $\alpha$ or the constant $C$ are measured or obtained from calibration measurements with a fluid of known high shear rheological properties. An example of such a high shear experiment is given in the top graph of Fig. 8. The viscosities as a function of high shear rates (filled symbols) are directly calculated from the hydrodynamic lubrication data of Fig. 8 via eqs. (40) and (41). The only adjustable parameter was the angle $\alpha$ that enters the geometry constant $C$, (which was for this specific annulus-on-plate geometry taken as $\alpha=1.6 \times 10^{-4} \mathrm{rad}$ as determined in the previous section). Although the high shear rate data in Fig. 8 are obtained without any knowledge about the fluid rheology, they give remarkably 
well the high shear rate response, not only for the quasi-Newtonian PDMS, but also for the shear thinning PIB in pristane solution up to shear rates of order $\mathrm{O}\left(10^{5}\right.$ $\left.\mathrm{s}^{-1}\right)$.

A general upper limit for the accessible shear rate range of this high shear rheometer was shown in Fig. 5 to be reached when the normal stresses arising in the fluid are the same as the applied normal force $F_{N}$ entering eq. (40) and (41). Although this limit is obviously not known for an unknown fluid, it can easily be tested when this limit is reached by varying the applied $F_{N}$. Furthermore, this limit can be increased by increasing $F_{N}$ within the capabilities of the rheometer.

\section{Conclusions}

Within this paper we have demonstrated that it is possible to directly obtain high shear rate flow curves from the sliding friction and angular velocity of an plateon-annulus triborheometrical geometry with known misalignment. For this we have introduced an analytical solution of Reynolds' equation for the hydrodynamic lubrication regime of the plate-of-annulus configuration. This solution indicates that the widening gap $h$ between the shearing surfaces can be directly related to a misalignment $\alpha$ between the surfaces and is proportional to the viscosity, angular velocity and inverse applied normal force (and thus the Gumbel number $G u$ ) with a characteristic power of $1 / 2$ or $1 / 3$, depending on the ratio of the gap to the misalignment $h /(\bar{R} \sin \alpha)$. For the triborheometer with its relatively well aligned surfaces and the generally observed case of $h \gg \bar{R} \sin \alpha$ the scaling of the effective gap $h \sim G u^{1 / 3}$ could be experimentally verified, as well as the resulting relation for the sliding friction $\mu=C^{\frac{1}{3}} G u^{\frac{2}{3}}$. Furthermore we have shown that the in literature reported linear relation $\mu: G u$ represents a case of hydrodynamic lubrication for which the lubrication forces are not yet sufficient to cause an effective widening of the shearing surface above an observed minimum effective gap. This minimum effective gap and misalignment angle determine a critical Gumbel number or sliding friction at which a transition from $\mu$ : $G u$ to $\mu \sim G u^{\frac{2}{3}}$ is (again also experimentally) observed. The analysis was expanded to a shear rate dependent viscosity model (the power-law model $\eta=K \dot{\gamma}^{n-1}$ ). The 
predicted scaling of the sliding friction with the angular velocity $\mu \sim h\left(K / F_{N}\right)^{2 /(n+2)} \Omega^{2 n /(n+2)}$ is again experimentally verified. Finally, the lubrication solution is used to formulate direct relations of viscosity as well as shear rate to the measured sliding friction or stress and angular velocity (eqs. (40) and (41)). These equations allow determining the shear rate dependent viscosities within the high shear rates of the hydrodynamic lubrication regime. Within the current paper the accessible shear rate regime was experimentally confirmed up to $\dot{\gamma}=10^{5} \mathrm{~s}^{-1}$.

\section{Acknowledgement}

This project was financially supported by the FWO - Vlaanderen (project G.0543.10N) and the Bijzonder Onderzoeksfonds K.U. Leuven (GOA 09/002). The author would like to thank Prof. H.P. Kavehpour and Prof. G.H. McKinley for fruitful discussions.

\section{Appendix A}

\section{Misalignment effects}

The alignment procedure of the bottom shearing surface parallel towards the top shearing surface described in the experimental section is still carrying forward the misalignment error of the top plate towards the axis of rotation of the rheometer. This remaining misalignment or tilt angle $\alpha$ is therefore maintained in both shearing surfaces (thus slightly different than the schematic in Fig. 2). While this difference has only a small effect on the theoretical lubrication analysis, it causes during one rotation cycle small variations of the gap height. This leads also to variations in the effectively applied normal force that the rheometer is trying to adjust (within the tolerance margin of $\pm 0.3 \mathrm{~N}$ ) to the set value in a feedback loop via small variations of the gap setting. A further result of the gap variation is that the measured torque $T$ or shear force $F_{s}$ is also showing an oscillation during a rotation cycle (as it can be seen for example in Fig. 9). The values of sliding friction in this report represent therefore an average over one or multiple full rotation cycles. It should be noted that although the oscillatory variation in the gap 
is undesired, it also provides a possibility for an independent determination of the magnitude of misalignment of the shearing surfaces and the tilt angle $\alpha$. In the boundary lubrication regime at low angular velocities the shearing surfaces are constantly in contact with each other during a full rotation of the upper shearing surface. For a single rotation the difference $\Delta h$ in Fig. 9 between the lowest gap readout of the rheometer (where the surfaces are parallel) and the largest gap readout (where the largest angle between the surface is observed) is related to the largest misalignment between the surfaces over a full rotation

$$
\Delta h=\bar{R} \sin \alpha
$$

For example from the gap variation in Fig. 9 the tilt angle obtained via eq. (42) is $\alpha=1.8 \times 10^{-4} \mathrm{rad}$. This experimental tilt angle can be compared to the theoretical tilt angles that are obtained from fitting eqs. (21) or (27) to experimental data of the hydrodynamic lubrication regime (with $\alpha$ as the only adjustable parameter). Fits of eqs. (21) and (27) yielded for example a value of $\alpha=3.8 \times 10^{-4} \mathrm{rad}$ in Fig. 5 and are thus of the same order of magnitude. The slightly higher value of the fitted effective gap can be due to a non-flatness of the surface. Such a non-flatness would not significantly be picked up by the observed $\Delta h$, but would lead to an additional lubrication flow that would reflect in the larger $\alpha$ of the fit.

\section{Figure 9 \\ Figure 10}

At higher angular velocities in the hydrodynamic lubrication regime the observed oscillations in the force signals become smaller (see Fig. 10) since the shearing surfaces are not in direct contact anymore, and since the effective gap $h_{\text {eff }}$ between the surfaces is increasing, making thus the relative gap error $\Delta h / h_{\text {eff }}$ smaller. Another difficulty that arises in the hydrodynamic lubrication regime can be seen in Fig. 10. Due to the small gap dimensions in comparison to the width of the annulus it takes a non-negligible time for the gap to approach a steady state value for a newly set angular velocity in the hydrodynamic lubrication regime. Since for such a reversed squeeze flow the rate of gap change $\mathrm{d} h / \mathrm{d} t$ is proportional to $1 / h^{3}$ (Baik et al. 2011) this effect becomes in particular important in the transitional 
lubrication regime where the top surface starts to "lift off" from the small initial gap separation.

\section{Temperature effects}

Viscous heating as a source for the observed shear thinning is a concern for high shear rate experiments (Dontula et al. 1999), but is in the current experiments negligible due to the narrow gap. The Nahme number as a dimensionless ratio of the time scales for thermal diffusion to viscous heating is for the current setup defined as $N a=\eta_{0} \beta h^{2} \dot{\gamma}^{2} / k T$, where $k$ is the thermal conductivity and $\beta$ is the thermal sensitivity $\beta=\left(T / \eta_{0}\right)|d \eta / d T|_{T=T_{0}}$ (Pipe et al. 2008). For PDMS with $k=$ $0.15 \mathrm{~W} / \mathrm{m} \mathrm{s}$ and $\beta=5.73$ at room temperature (Van Hemelrijck 2005) the Nahme number is even for the highest viscosities and rates in the hydrodynamic lubrication regime of order $\mathrm{O}\left(10^{-2}\right)$ and viscous heating can therefore be neglected. Similar, for the PIB in pristane solution with $k=0.12 \mathrm{~W} / \mathrm{m} \mathrm{s}$ and $\beta \sim$ 9.8 (Schultheisz and Leigh 2002) the Nahme number remains even for the highest rate of $\dot{\gamma}=10^{5} \mathrm{~s}^{-1}$ with $\mathrm{Na}=4 \times 10^{-2}$ sufficiently below unity. The negligible effect of viscous heating were also experimentally confirmed with a thermocouple. For all experiments reported here the temperature of the samples was confirmed to remain constant.

However, a problem is arising from an uncontrollable heating of the rheometer head that houses the bearings of the rotational shaft. During long-time tests at higher shearing stresses the temperature of the head and also of the shaft holding the upper shearing plate is noticeably increasing. This causes an expansion of the shaft which is, however, not directly affecting the triborheometrical experiment. Although the expansion of the shaft is initially leading to a decreasing gap between the shearing surfaces, this is also increasing the effectively applied normal forces. As the rheometer is adjusting this normal force increase by raising the upper plate, the gap between the shearing surfaces is staying constant and is thus independent of the shaft expansion (though with the additional noise that is originating from the feedback loop of the normal force control). However, although the absolute gap distance is staying constant, the apparent gap distance reported by the rheometer (which is the effective position of the rheometer head) includes the (unknown) expansion of the shaft. This is for example clearly visible in Fig. 10 where the torque $T$ is acquiring a steady state within the first $200 \mathrm{~s}$, whereas the apparent gap is continuous increasing over the course of the 
experiment. This uncontrolled expansion of the rotational shaft hinders therefore a correlation of the rheometer readout of an apparent gap to the actual gaps between the shearing surfaces.

\section{Inertial effects}

High shear rates in a parallel plate setup can lead to secondary flows and a loss of sample due to inertial effects. Such centrifugal effects can no longer be ignored when $0.15 \rho \bar{R}^{2} \Omega^{2} / \gamma$ becomes larger then unity (Connelly and Greener 1985), where $\gamma$ is the surface tension of the liquid and $\rho$ is the density. However, even for the highest shear rates and largest shearing gaps reached in the hydrodynamic lubrication regime this value was at least an order of magnitude below unity and inertial effects can thus be neglected.

\section{Pressure effects}

The highest average additional pressure that is applied to the sample by the normal load is of order $\mathrm{O}(0.1 \mathrm{bar})$, therefore the effects of pressure on the viscosity are negligible and the fluids can be treated as isoviscous in the hydrodynamic lubrication regime

\section{References}

Andablo-Reyes, E., J. de Vicente and R. Hidalgo-Alvarez, "On the nonparallelism effect in thin film plate-plate rheometry". Journal of Rheology 55, 981-986 (2011a).

Andablo-Reyes, E., R. Hidalgo-Alvarez and J. de Vicente, "A method for the estimation of the film thickness and plate tilt angle in thin film misaligned plateplate rheometry". Journal of Non-Newtonian Fluid Mechanics 165, 1419-1421 (2010).

Andablo-Reyes, E., R. Hidalgo-Alvarez and J. de Vicente, "Controlling friction using magnetic nanofluids". Soft Matter 7, 880-883 (2011b).

Baik, S. J., P. Moldenaers and C. Clasen, "A sliding plate microgap rheometer for the simultaneous measurement of shear stress and first normal stress difference". Review of Scientific Instruments 82, 035121 (2011).

Bishop, A. I., T. A. Nieminen, N. R. Heckenberg and H. Rubinsztein-Dunlop, "Optical microrheology using rotating laser-trapped particles". Physical Review Letters 92, 4 (2004).

Bombard, A. J. F. and J. de Vicente, "Thin-Film Rheology and Tribology of Magnetorheological Fluids in Isoviscous-EHL Contacts". Tribology Letters 47, 149-162

Boskovic, S., J. W. M. Chon, P. Mulvaney and J. E. Sader, "Rheological measurements using microcantilevers". Journal of Rheology 46, 891-899 (2002). Cagnon, M. and G. Durand, "Mechanical Shear of Layers in Smectic-A and Smectic-B Liquid-Crystal". Physical Review Letters 45, 1418-1421 (1980). 
Clasen, C., "Determining the true slip of a yield stress material with a sliding plate rheometer". Rheologica Acta 51, 883-890 (2012).

Clasen, C., B. P. Gearing and G. H. McKinley, "The flexure-based microgap rheometer (FMR)". Journal of Rheology 50, 883-905 (2006).

Clasen, C., H. P. Kavehpour and G. H. McKinely (2007). From

bulkmircorheology to tribology. The Society of Rheology $79^{\text {th }}$ Annual Meeting.

Salt Lake City, UT, USA,

Clasen, C., H. P. Kavehpour and G. H. McKinley, "Bridging Tribology And

Microrheology Of Thin Films". Applied Rheology 20, 196-208 (2010).

Clasen, C. and G. H. McKinley, "Gap-dependent microrheometry of complex

liquids". Journal of Non-Newtonian Fluid Mechanics 124, 1-10 (2004).

Connelly, R. W. and J. Greener, "High-Shear Viscometry with a Rotational

Parallel-Disk Device". Journal of Rheology 29, 209-226 (1985).

Crassous, J. J., R. Regisser, M. Ballauff and N. Willenbacher, "Characterization of the viscoelastic behavior of complex fluids using the piezoelastic axial vibrator". Journal of Rheology 49, 851-863 (2005).

Crocker, J. C., M. T. Valentine, E. R. Weeks, T. Gisler, P. D. Kaplan, A. G. Yodh and D. A. Weitz, "Two-point microrheology of inhomogeneous soft materials".

Physical Review Letters 85, 888-891 (2000).

Davies, G. A. and J. R. Stokes, "Thin film and high shear rheology of multiphase complex fluids". Journal of Non-Newtonian Fluid Mechanics 148, 73-87 (2008). de Vicente, J., H. A. Spikes and J. R. Stokes, "Viscosity ratio effect in the emulsion lubrication of soft EHL contact". Journal of Tribology-Transactions of the Asme 128, 795-800 (2006).

de Vicente, J., J. R. Stokes and H. A. Spikes, "The frictional properties of newtonian fluids in rolling-sliding soft-EHL contact". Tribology Letters 20, 273286 (2005).

Dhinojwala, A. and S. Granick, "Micron-gap rheo-optics with parallel plates". Journal of Chemical Physics 107, 8664-8667 (1997).

Dontula, P., C. W. Macosko and L. E. Scriven, "Does the viscosity of glycerin fall at high shear rates?". Industrial \& Engineering Chemistry Research 38, 17291735 (1999).

Erni, P., M. Varagnat, C. Clasen, J. Crest and G. H. McKinley, "Microrheometry of sub-nanolitre biopolymer samples: non-Newtonian flow phenomena of carnivorous plant mucilage". Soft Matter 7, 10889-10898 (2011).

Ewoldt, R. H., C. Clasen, A. E. Hosoi and G. H. McKinley, "Rheological fingerprinting of gastropod pedal mucus and synthetic complex fluids for biomimicking adhesive locomotion". Soft Matter 3, 634-643 (2007). Fritz, G., W. Pechhold, N. Willenbacher and N. J. Wagner, "Characterizing complex fluids with high frequency rheology using torsional resonators at multiple frequencies". Journal of Rheology 47, 303-319 (2003).

Gittes, F., B. Schnurr, P. D. Olmsted, F. C. MacKintosh and C. F. Schmidt, "Microscopic viscoelasticity: Shear moduli of soft materials determined from thermal fluctuations". Physical Review Letters 79, 3286-3289 (1997).

Gong, J. P., G. Kagata and Y. Osada, "Friction of gels. 4. Friction on charged gels". Journal of Physical Chemistry B 103, 6007-6014 (1999).

Heyer, P. and J. Lauger, "Correlation between friction and flow of lubricating greases in a new tribometer device". Lubrication Science 21, 253-268 (2009). Kagata, G., J. P. Gong and Y. Osada, "Friction of gels. 6. Effects of sliding velocity and viscoelastic responses of the network". Journal of Physical Chemistry B 106, 4596-4601 (2002). 
Kagata, G., J. P. Gong and Y. Osada, "Friction of gels. 7. Observation of static friction between like-charged gels". Journal of Physical Chemistry B 107, 1022110225 (2003).

Kavehpour, H.P. and G. H. McKinley, "Triborheometry from Gap-Dependent Rheology to Tribology". Tribology Letters 17, 327-335 (2004).

Kojic, N., J. Bico, C. Clasen and G. H. McKinely, "Ex-Vivo Rheology of Spider Silk". Journal of Experimental Biology 209, 4355-4362 (2006).

Kramer, J., J. T. Uhl and R. K. Prudhomme, "Measurement of the Viscosity of Guar Gum Solutions to 50,000 1/s Using a Parallel Plate Rheometer". Polymer Engineering and Science 27, 598-602 (1987).

Levine, A. J. and T. C. Lubensky, "One- and two-particle microrheology". Physical Review Letters 85, 1774-1777 (2000).

Macosko, C., Rheology: Principles, Measurements, and Applications, VCH Publishers, New York (1994).

Mason, T. G., K. Ganesan, J. H. vanZanten, D. Wirtz and S. C. Kuo, "Particle tracking microrheology of complex fluids". Physical Review Letters 79, 32823285 (1997).

Montfort, J.P., A. Tonck, J.L. Loubet and J.M. Georges, "Microrheology of highpolymer solutions". Journal of Polymer Science, Part B: Polymer Physics 29, 677-682 (1991).

Mukhopadhyay, A. and S. Granick, "Micro- and nanorheology". Current Opinion in Colloid \& Interface Science 6, 423-429 (2001).

Oogaki, S., G. Kagata, T. Kurokawa, S. Kuroda, Y. Osada and J. P. Gong, "Friction between like-charged hydrogels-combined mechanisms of boundary, hydrated and elastohydrodynamic lubrication". Soft Matter 5, 1879-1887 (2009). Pine, D. J., D. A. Weitz, P. M. Chaikin and E. Herbolzheimer, "Diffusing-Wave Spectroscopy". Physical Review Letters 60, 1134-1137 (1988).

Pipe, C. J., T. S. Majmudar and G. H. McKinley, "High shear rate viscometry". Rheologica Acta 47, 621-642 (2008).

Popescu, G., A. Dogariu and R. Rajagopalan, "Spatially resolved microrheology using localized coherence volumes". Physical Review E 65, art. no. 041504 (2002).

Schultheisz, C.R. and S.J. Leigh (2002). Certification of the rheological behavior of SRM 2490, polyisobutylene dissolved in 2,6,10,14-tetramethylpentadecane. Gaithersburg, National Institute of Standards and technology special publication 262-143.

Soga, I., A. Dhinojwala and S. Granick, "Optorheological studies of sheared confined fluids with mesoscopic thickness". Langmuir 14, 1156-1161 (1998). Vadillo, D., W. Mathues and C. Clasen, "Microsecond relaxation processes in shear and extensional flows of weakly elastic polymer solutions". Rheologica Acta 51, 755-769 (2012).

Van Hemelrijck, E. (2005). Effect of Physical Compatibilzation on the Morphology of Immiscible Polymer Blends. Chemical Engineering. Leuven, Belgium, Katholieke Universiteit Leuven. PhD: 170.

Vananroye, A., P. Leen, P. Van Puyvelde and C. Clasen, "TTS in LAOS:

Validation of Time Temperature Superposition under Large Amplitude

Oscillatory Shear". Rheologica Acta 50, 795-807 (2010).

Waigh, T. A., "Microrheology of complex fluids". Reports on Progress in Physics 68, 685-742 (2005).

Williams, J., Engineering Tribology, Cambridge University Press, New York (2005). 
Xu, J., S. Costeux, J. M. Dealy and M. N. De Decker, "Use of a sliding plate rheometer to measure the first normal stress difference at high shear rates". Rheologica Acta 46, 815-824 (2007). 


\section{Figures}

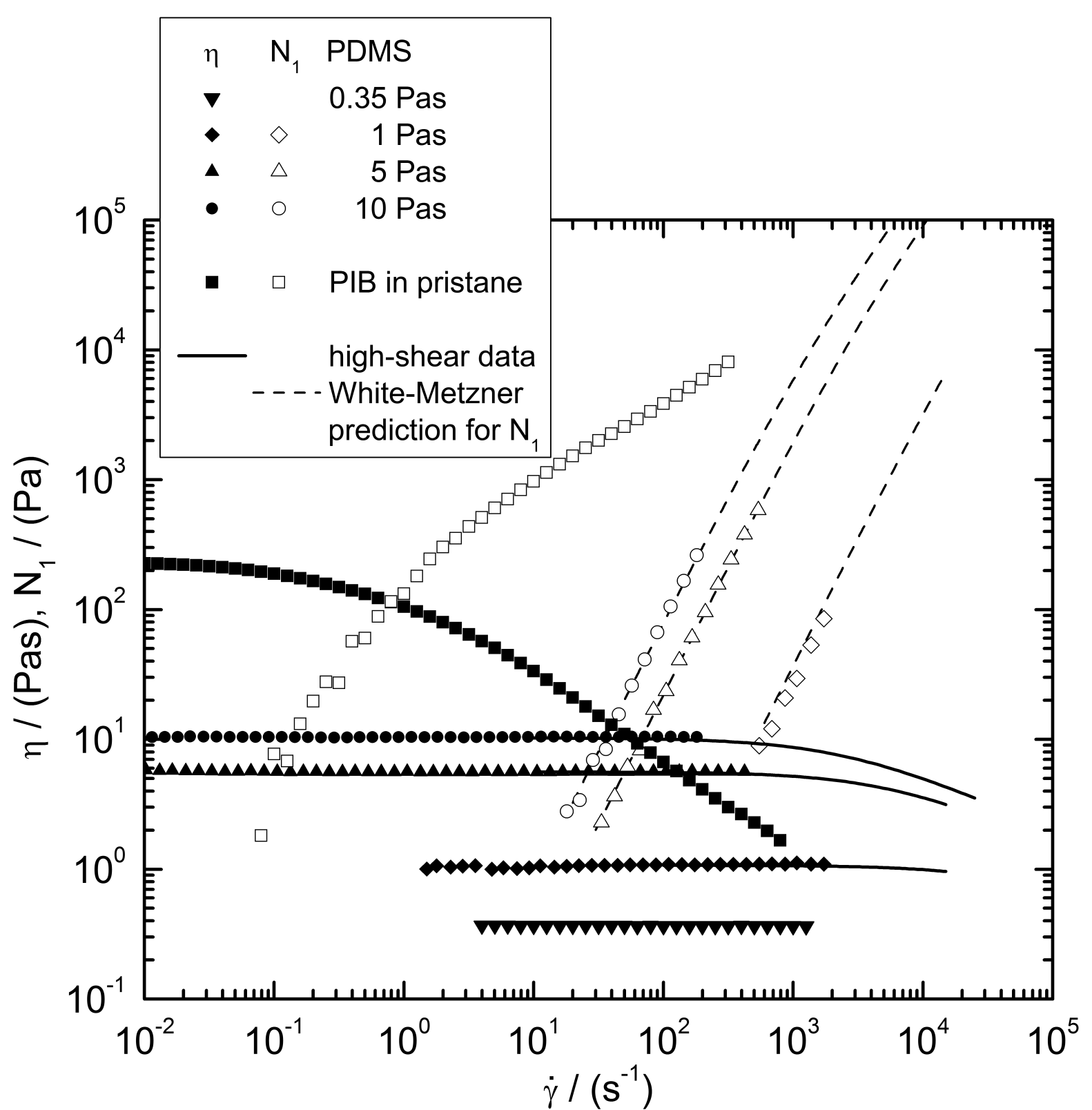

Figure 1 Viscosity $\eta$ (filled symbols) and first normal stress difference $\mathrm{N}_{1}$ (open symbols) as function of the shear rate or four PDMS oils of different molecular weights and viscosities and a shear thinning solution of polyisobutylene (PIB) in pristane. Solid lines represent measurements at higher rates of selected fluids with a FMR thin film rheometer, and dashed lines represent predictions of $\mathrm{N}_{1}$ at higher rates from the White-Metzner model (data taken from (Clasen et al. 2010)). 

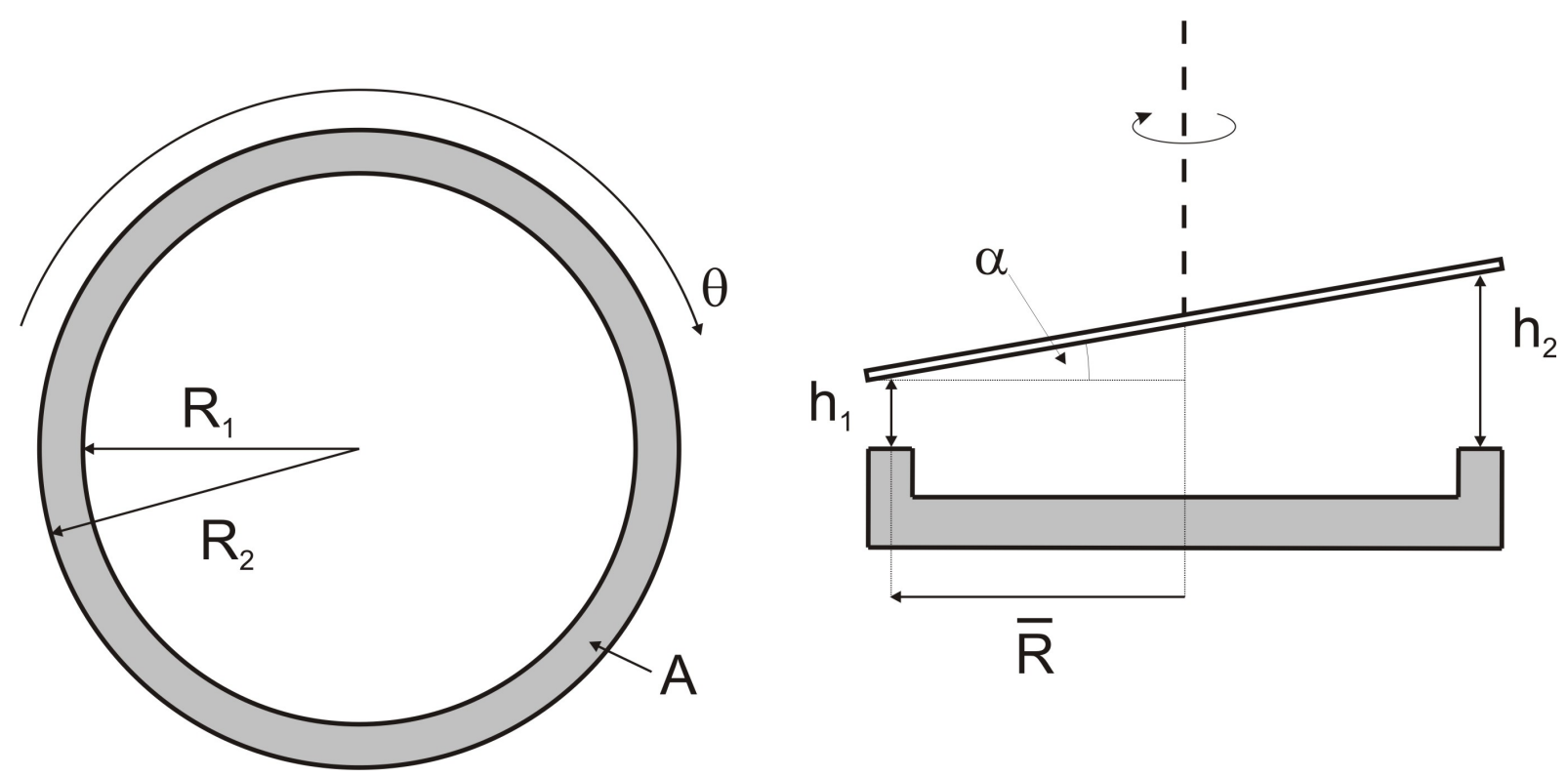

Figure 2 Schematic of the plate-on annulus geometry used for the triborheometrical experiments. The top plate is mounted to the rotational shaft of the rheometer while the bottom annulus is fixed to the peltier element.

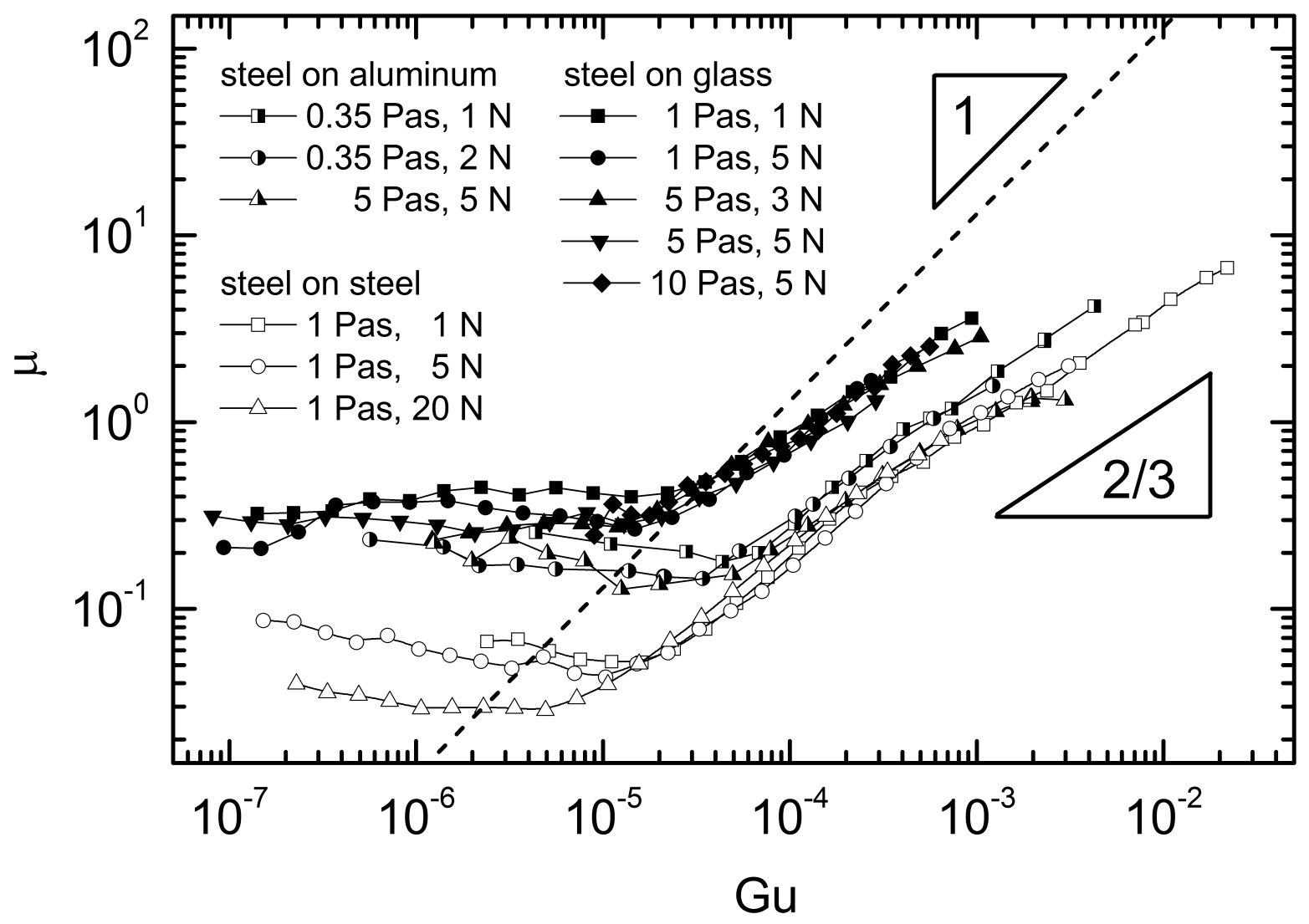

Figure 3 Stribeck curves - non-dimensional sliding friction $\mu$ as a function of the non-dimensional rotation speed $G u$ for the PDMS melts of different viscosities, shearing surfaces and applied normal loads (indicated in the legend). For comparison the dashed line represents the theoretical curve for $\mu$ as function of $G u$ obtained from eq. (9) for the sample of $1 \mathrm{~Pa} s$ in the steel on glass surface combination (using a constant average gap $h=2 \mu \mathrm{m})$. 


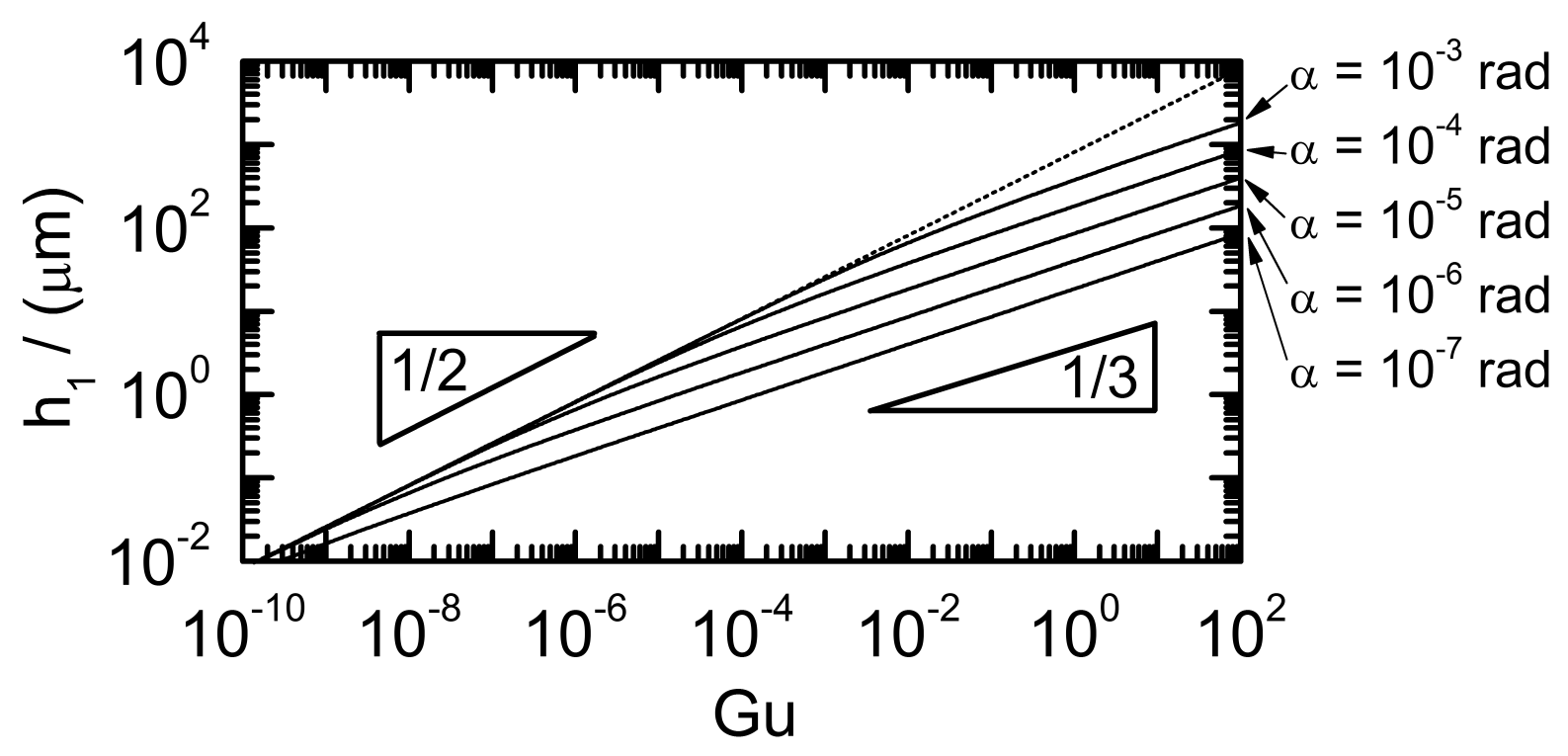

Figure 4 Theoretical gap $h_{1}$ as function of the Gumbel number $G u$ for different tilt angles $\alpha$ following eq. (19) and a geometrical constant $C \sin \alpha=428.54$ as calculated for the glass/steel geometry. 


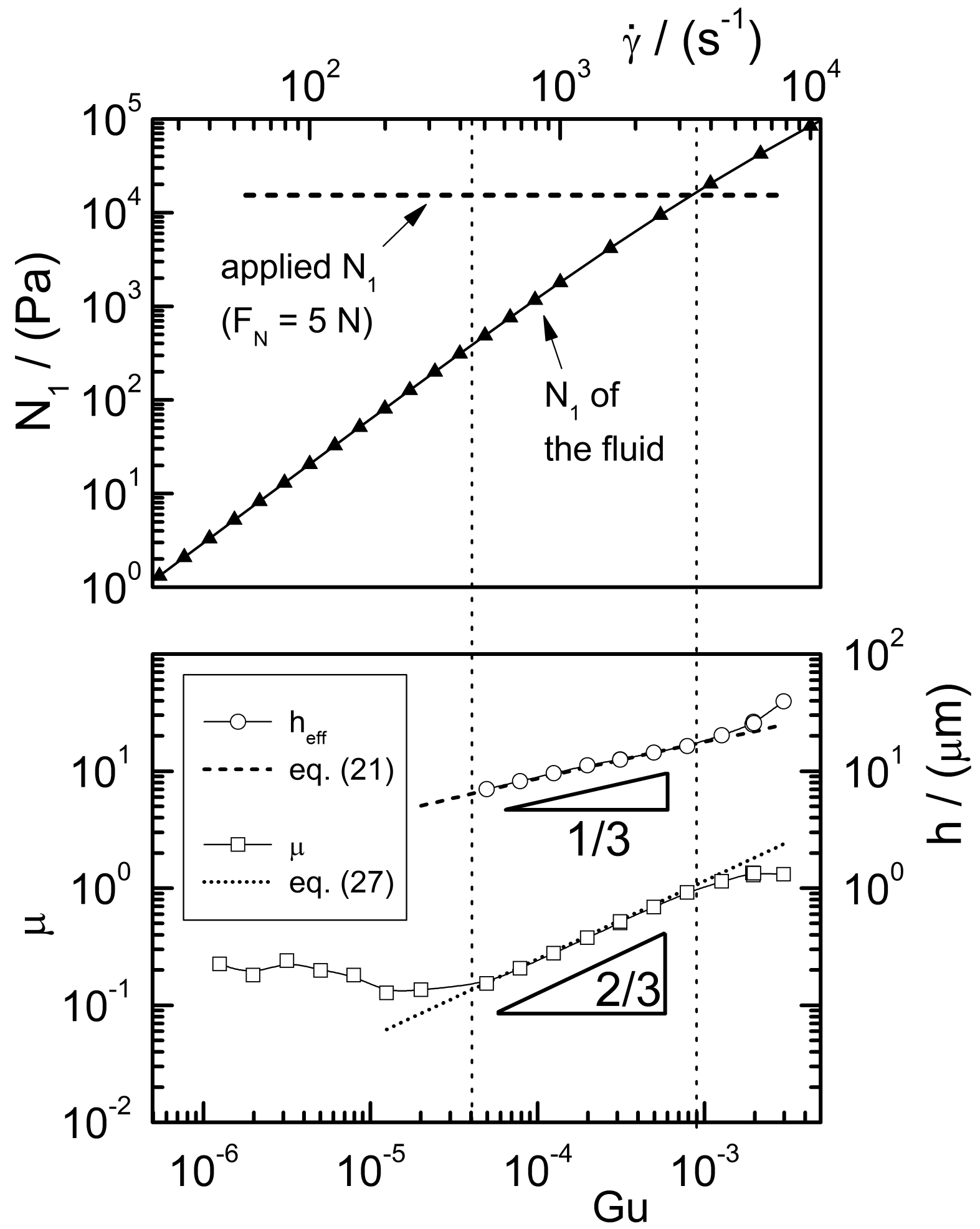

Figure 5 Sliding friction $\mu$ and the gap $h_{\text {eff }}$ in the hydrodynamic lubrication regime as a function of the Gumbel number $G u$ for a PDMS melt with a viscosity of 5 Pas measured with the aluminum/steel shearing surface combination at a constant applied normal load of $F_{N}=5 \mathrm{~N}$. The dashed line is a fit of the gap with eq. (21), and the dotted line is a fit of $\mu$ with eq. (27). Both fits are done with the tilt angle $\alpha$ as the only fitting parameter, resulting in $\alpha=3.8 \times 10^{-4} \mathrm{rad}$. The upper graph gives in addition t to the applied normal load also $N_{1}$ of the fluid itself as a function of the shear rate $\dot{\gamma}$. The shear rate axis is scaled via eq. (21) to match the Gumbel number axis in the hydrodynamic lubrication regime 


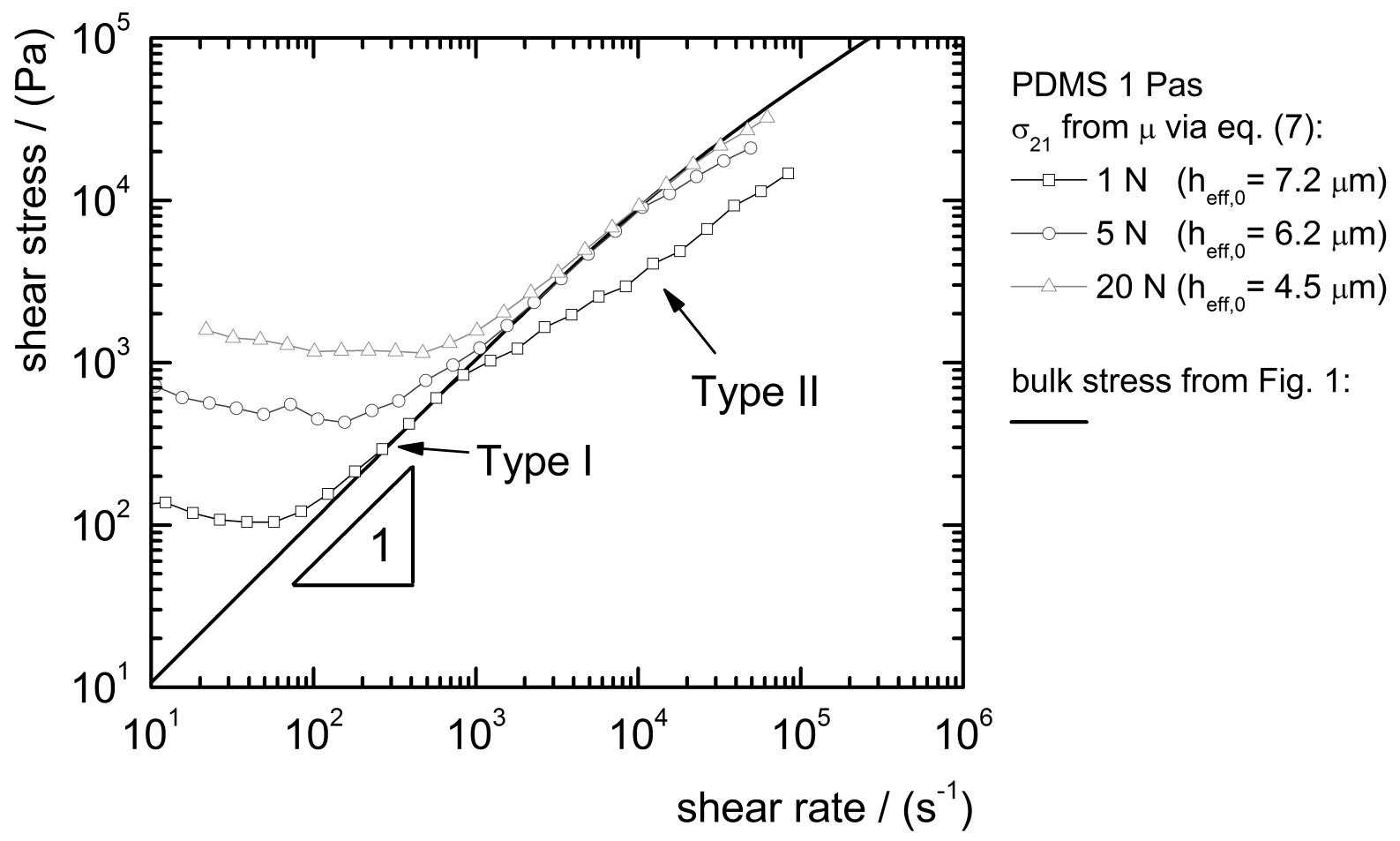

Figure 6 Shear stresses of the triborheological measurements as a function of an apparent shear rate for PDMS with a nominal viscosity of 1 Pas using the steel/steel shearing surface combination. The required constant effective gaps $h_{\text {eff }, 0}$ to shift the rates $\dot{\gamma}=\Omega \bar{R} / h_{\text {eff }}$ onto the bulk flow curve (straight line) are given in brackets.

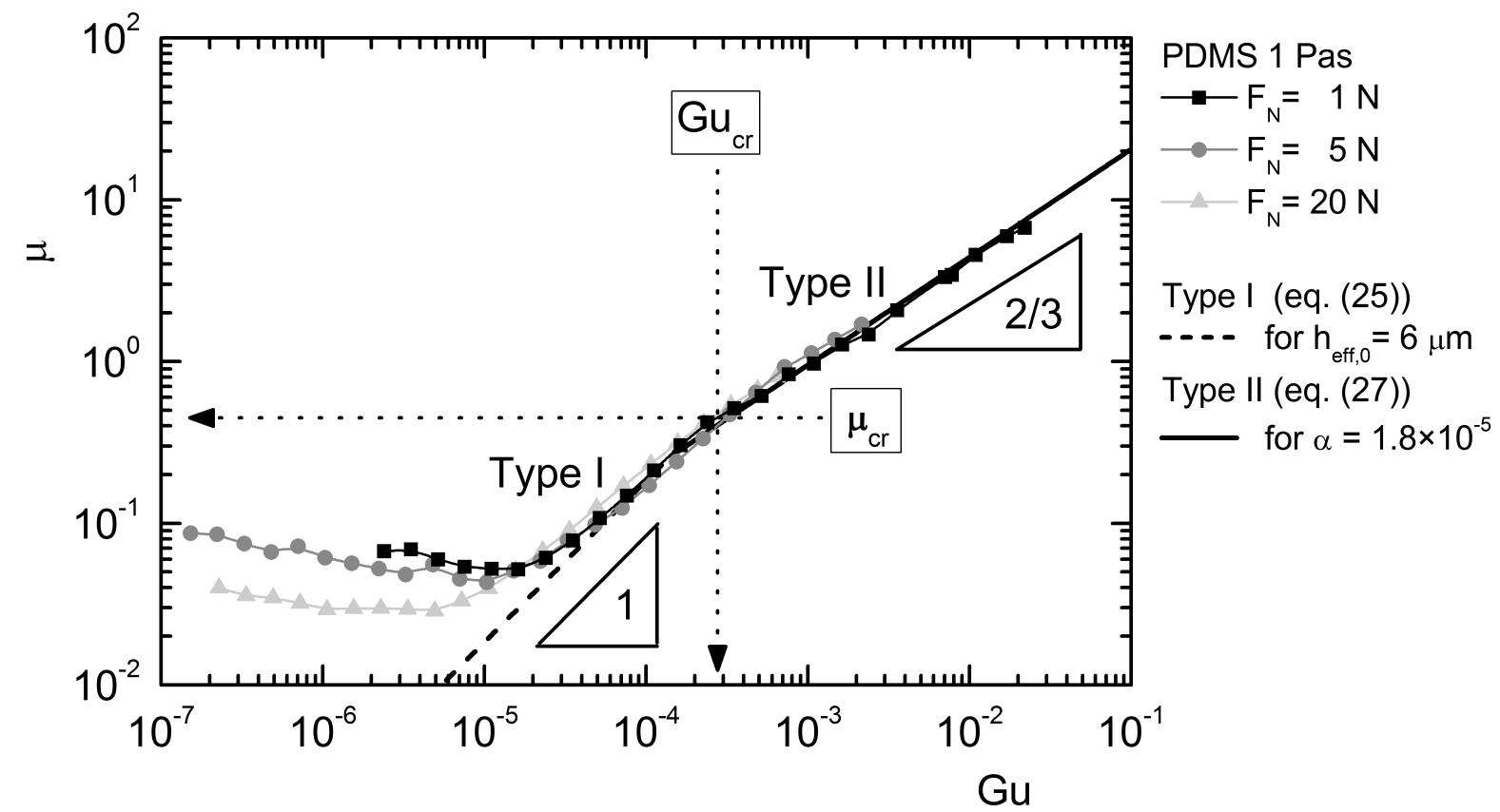

Figure $7 \quad$ Friction coefficient $\mu$ as a function of the Gumbel number $G u$ for the PDMS sample of Fig. 6 at different applied normal loads $F_{N}$. The dashed line indicates the hydrodynamic lubrication type I of eq. (25), for a minimum effective gap of $h_{e f f, 0}=6 \mu \mathrm{m}$. The straight line represents the hydrodynamic lubrication type II of eq. (27) for a tilt angle of $\alpha=1.8 \times 10^{-5} \mathrm{rad}$. 


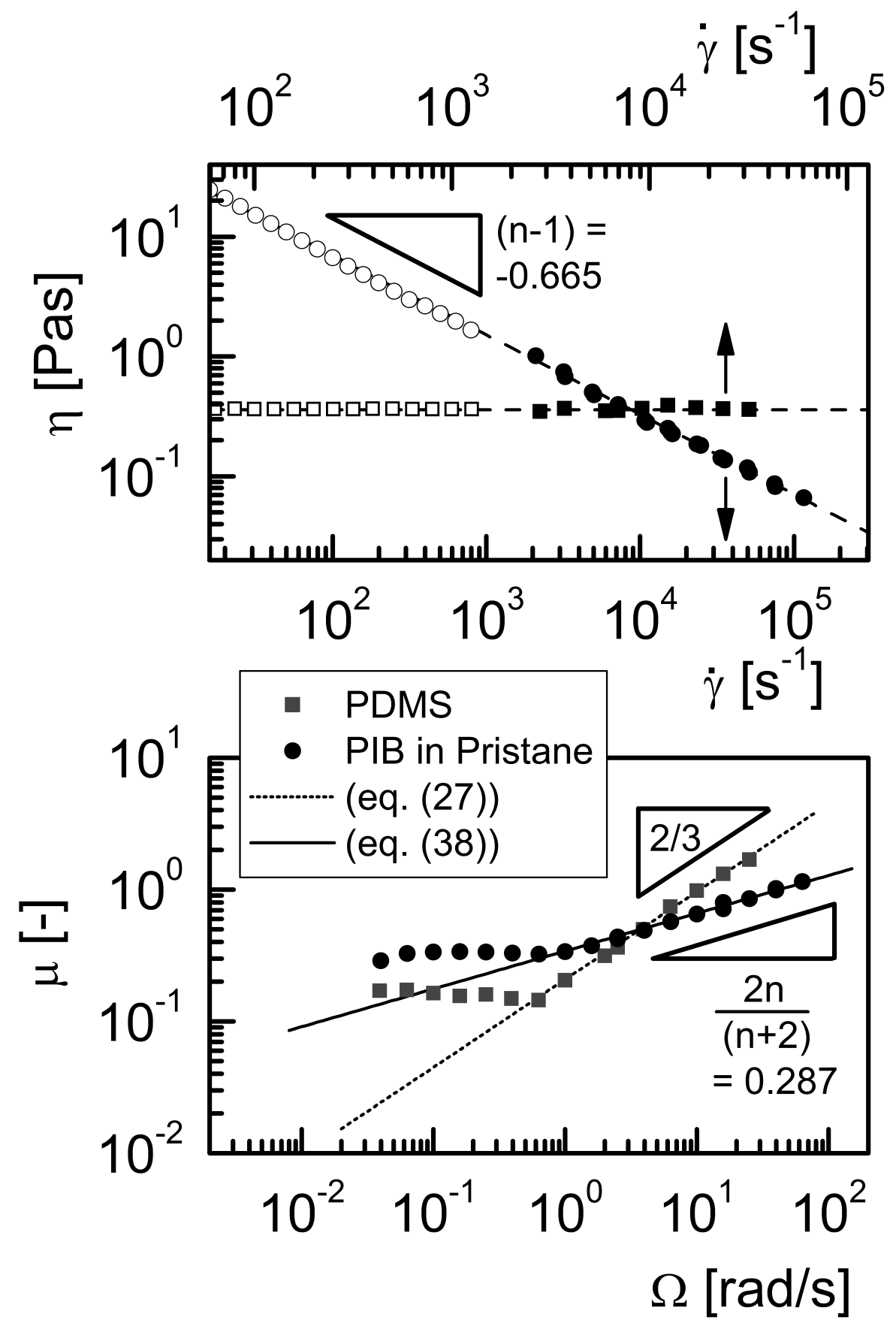

Figure $8 \quad$ Sliding friction $\mu$ as a function of the angular velocity $\Omega$ for a PDMS melt and a shear thinning solution of polyisobutylene in pristane, both measured with the same aluminum/steel shearing surface combination with a normal load of $2 \mathrm{~N}$. The hydrodynamic lubrication regimes are fitted with eq. (27) and eq. (38) respectively, resulting in tilt angles as the fitting parameter of $\alpha=1.90 \times 10^{-4} \mathrm{rad}$ and $\alpha=1.86 \times 10^{-4} \mathrm{rad}$. High shear rate viscosity data and shear rates (filled symbols) in the upper graph are directly calculated from the sliding friction of the lower graph via eqs. (40) and (41). 


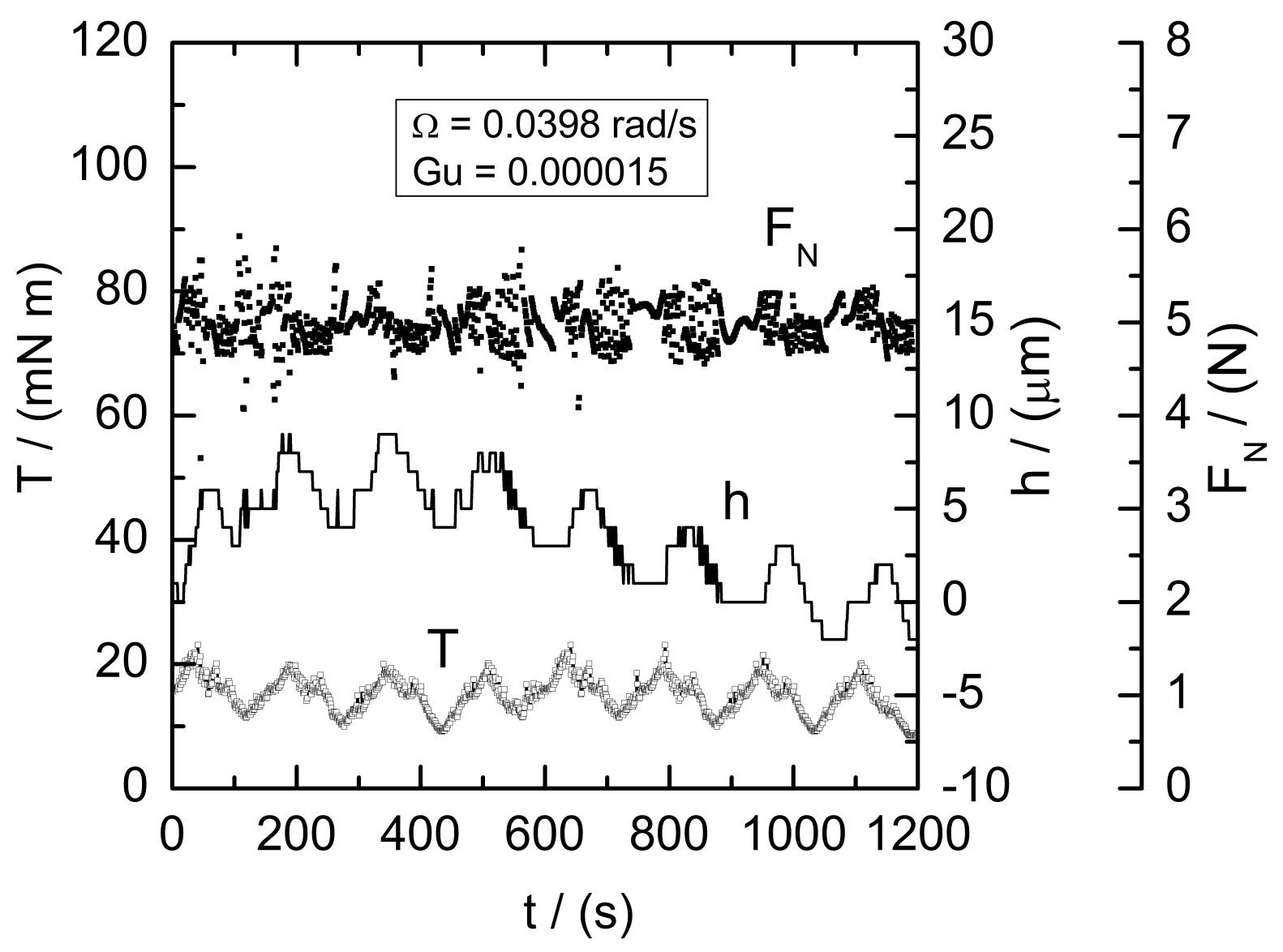

Figure $9 \quad$ Evolution of sliding friction $\mu$ (open symbols), applied normal force $F_{N}$ (closed symbols), and apparent gap $h$ (straight line) with time $t$ for a PDMS melt of nominal viscosity 5 Pas. The data is measured with the aluminum/steel surface combinations within the boundary lubrication regime at constant angular velocities of $\Omega=1.5 \times 10^{-4} \mathrm{rad} / \mathrm{s}$. 


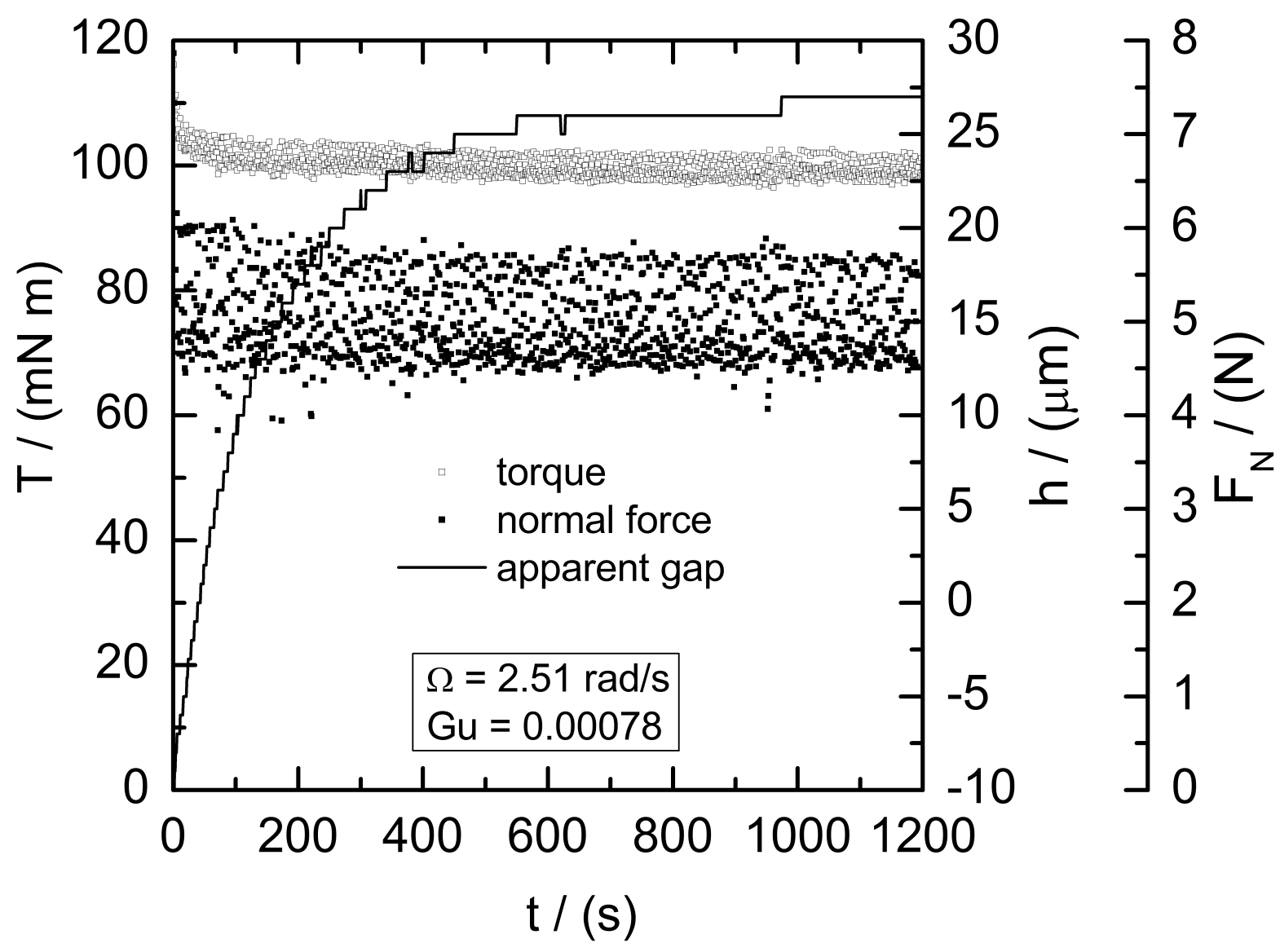

Figure $10 \quad$ Evolution of sliding friction $\mu$ (open symbols), applied normal force $F_{N}$ (closed symbols), and apparent gap $h$ (straight line) with time $t$ for the same PDMS sample and geometry as in Fig. 9 , but within the hydrodynamic lubrication regime at constant angular velocities of $\Omega=2.51 \mathrm{rad} / \mathrm{s}$. 